\title{
Stress Effects on Vibrational Spectra of a Cubic Hybrid Perovskite: A Probe of Local Strain
}

\author{
Kuntal Talit and David A. Strubbe* \\ Department of Physics, University of California, Merced, 5200 N. Lake Rd., Merced, CA \\ 95343 \\ E-mail: dstrubbe@ucmerced.edu
}




\begin{abstract}
Inhomogeneous strain may develop in hybrid organic metal-halide perovskite thin films due to thermal expansion mismatch with a fabrication substrate, polycrystallinity or even light soaking. Measuring these spatially varying strains is difficult but of prime importance for understanding the effects on carrier mobility, non-radiative recombination, degradation and other optoelectronic properties. Local strain can be mapped using the shifts in vibrational frequencies using Raman or infrared microscopy. We use density functional theory to investigate the effect of uniaxial strain on the vibrations of pseudo-cubic methylammonium lead iodide $\left(\mathrm{CH}_{3} \mathrm{NH}_{3} \mathrm{PbI}_{3}\right)$, and identify the vibrational modes most favorable for local strain mapping $\left(86 \mathrm{~cm}^{-1}, 97 \mathrm{~cm}^{-1}, 1457\right.$ $\mathrm{cm}^{-1}$, and $1537 \mathrm{~cm}^{-1}$ ) and provide calibration curves. We explain the origin of the frequency changes with strain using dynamical matrix and mode eigenvector analysis and study strain-induced structural changes. We also calculate mode Grüneisen parameters, giving information about anharmonicity and anisotropic negative thermal expansion as recently reported for other phases. Our results provide a basis for strain mapping in hybrid perovskites to further the understanding and control of strain, and improve stability and photovoltaic performance.
\end{abstract}

\title{
1 Introduction
}

Organic-inorganic hybrid perovskites are promising materials for next-generation solar cell applications. $\underline{\underline{1}}$ They have a direct bandgap, $\underline{\underline{2}, \underline{3}}$ high absorption coefficient $\underline{\underline{4}}$ long diffusion length, $\underline{\underline{5}, \underline{6}}$ and large carrier mobility $\underline{\underline{7}}$ which make them favorable for PV applications. In the last 10 years the record photoconversion efficiency (PCE) has increased drastically from $3.8 \% \underline{8}$ to $25.2 \% . \underline{9}$ Owing to other benefits like ease of bandgap engineering, $\underline{10,11}$ flexibility for use in portable electronic devices, $\underline{12}$ tunability of transparency to light for tandem cells, $\underline{13}$ and suitability for mass production $\frac{14}{}$ with a cheaper cost, perovskites have been the object of great interest in the solar cell industry recently. There are also other non-PV applications of 
perovskites, e.g. spin-optoelectronic applications, $\underline{\underline{15}}$ luminescent solar concentrators, $\underline{\underline{16}}$ and light-emitting diodes. $\underline{\underline{17}}$

Despite these favorable properties, hybrid perovskites suffer from serious instability due to moisture, $\underline{\underline{18}}$ heat $\underline{\underline{19}}$ and light, $\underline{\underline{20}}$ which hinder their commercialization as solar cells. Although different encapsulation techniques can help to eliminate the moisture issue, $, \underline{21}, \underline{22}$ degradation due to unavoidable exposure to heat and light is still a challenge that needs to be addressed. Different studies have suggested that strain plays an important role in both degradation and photovoltaic performance of perovskites. $\underline{23} \underline{\underline{32}}$ This strain arises in thin films via substrate thermal expansion mismatch, epitaxial lattice mismatch, $\underline{\underline{33}}$ phase transitions, grain structure, and composition inhomogeneities, creating both global and local strain. $\underline{\underline{30}}$ Strain within the lattice can affect the carrier dynamics, $\underline{\underline{31}}$ defect concentration, non-radiative recombination, $\underline{\underline{30}}$ bandgap $\underline{27}$ etc. and decrease the PCE of the device. Strain can also decrease the activation energy of ion migration within the material which accelerates its degradation..$\underline{25}$ Slow photocurrent degradation caused by deep trap states has been attributed to polarons, consisting of strain and reorientation of the organic ion, which form under light soaking. $\underline{\underline{24}}$ On the other hand, light soaking can also create lattice expansion which reduces residual strain within the lattice and help to increase efficiency. $\underline{\underline{29}}$ High compressive strain (more than $1 \%$ for cubic $\mathrm{CH}_{3} \mathrm{NH}_{3} \mathrm{PbI}_{3}$ ) can also be responsible for structural phase changes. $\underline{\underline{26}} \underline{\underline{27}}$ To understand all these strain-induced behaviors, we need to understand the structural and vibrational changes due to strain.

In this work, we study as a benchmark system the pseudo-cubic phase of methylammonium lead iodide (MAPI, $\mathrm{CH}_{3} \mathrm{NH}_{3} \mathrm{PbI}_{3}$ ), $\underline{34}, \frac{35}{4}$ one of the best studied hybrid perovskites. Depending on temperature, MAPI exists in three different phases: orthorhombic at low temperature $(T<161.4 \mathrm{~K})$, tetragonal at room temperature $(161.4 \mathrm{~K}<T<330.4 \mathrm{~K})$ and cubic (or pseudo-cubic) ${ }^{\frac{36}{6}}$ at high temperature $(T>330.4 \mathrm{~K})$. Both orthorhombic and tetragonal structures are based on $\sqrt{2} \times \sqrt{2} \times 2$ supercells of the cubic structure with four times the number of atoms in the unit cell. 
We have chosen to begin with a study of the high-temperature cubic phase due to its simpler structure. Further work will study the orthorhombic and tetragonal structures, which clearly have their own individual characteristics. The cubic phase can be considered as a reference to describe the structures of the other phases. $\frac{36}{}$ It is not only significant at high temperatures, but also it can be stabilized at lower temperature in quantum dots via ligands on the surface,,$^{37}$ use of other organic cations ${ }^{38}$ such as formamidinium, or substitution of $\mathrm{Br}$ for I. $\underline{39}$ Generally cubic phases are found to have higher PCE than the other phases. $\underline{40}$ Due to the strong resemblance between the cubic, tetragonal, and orthorhombic phases' vibrational properties, particularly in the mid-frequency range, $\underline{\underline{41}}$ we believe this work also gives insight into those phases.

Vibrational properties of all three phases have been extensively studied. $\stackrel{41-49}{\underline{49}}$ The infrared (IR) and Raman spectra of all three phases show three distinct regimes of the vibrational frequencies, due to the Pb-I cage and coupled cage/methylammonium (MA) ion modes at low frequency, and MA ion at medium and high frequency. $\underline{47}$ There are only a few experimental results on vibrational spectroscopy available for the cubic structure. $\underline{42}, \underline{43}, \underline{45}, 49$ Others have argued that there is no Raman activity of the low-frequency $\mathrm{PbI}$ cage modes for cubic MAPI $\underline{50}$ or cubic $\mathrm{MAPbBr}_{3}, \underline{\underline{51}}$ based on the symmetry argument given for $\mathrm{CsPbCl}_{3}$ and $\mathrm{SrTiO}_{3} \cdot{ }^{36}$ Observation of small but nonzero Raman activity in this regime was attributed to disorder. $\underline{50}$ However, even in a perfectly ordered cubic MAPI crystal the fact that we have different atomic coordinates from $\mathrm{CsPbCl}_{3}$ means that the $\mathrm{PbI}$ cage phonon modes (by hybridization with the MA ion modes) can have other irreducible representations that are Raman active, as indeed we demonstrate in this work. Measurement of low-frequency Raman has been attributed to light-induced degradation products, $\underline{50}$ but our results suggest the validity of at least some measurements in this regime, demonstrated by the agreement in frequencies in Fig. 2.

The detailed behavior of vibrational modes under applied tensile and compressive strain for these materials has not been studied. A high-pressure study of $\mathrm{MAPbBr}_{3} \frac{51}{}$ showed only 
broad features in the cubic phase in the low-frequency region, and clear peaks occurring after phase transition to tetragonal. However the related macroscopic Grüneisen parameter $\gamma$, and the related thermal expansion, have been studied. For the tetragonal structure, a negative thermal expansion coefficient was measured along the [001] direction (c-axis). $\underline{52,53}$ For the cubic structure, the Grüneisen parameter was calculated and measured in previous studies $\underline{41.52}$ but not along all three crystallographic axes. $\gamma$ (including the individual mode Grüneisen parameters, also known as phonon deformation potentials $\underline{\underline{54}}$ ) are of fundamental interest as a probe of anharmonicity, which is quite strong in perovskites $\underline{55}, \underline{56}$ and relates to phonon-phonon scattering and thermal transport.

Using strain effects on vibrations to measure local strain can help us to understand the material degradation and its performance instability. To measure local strain within a material, we want to focus on a length scale $\sim \mu \mathrm{m}$ or below. Most of the experiments done so far to measure strain in perovskites have used grazing incidence X-ray diffraction (GIXRD), normal XRD $\underline{25}, \underline{29}, 31,57$ or the substrate curvature method $\underline{57}$ which probe large areas. Scanning XRD has been used for more local mapping, but requires a synchrotron. $\underline{\underline{30}}$ Another standard non-destructive technique used to characterize perovskites and other semiconductor thin films is Raman spectroscopy. $\underline{\underline{58}}$ Raman microscopy, in which small areas are probed, can be used to measure the stress distribution within a material. This is a well established technique for crystalline $\mathrm{Si}(\mathrm{c}-\mathrm{Si})$ in the semiconductor industry, $\underline{\underline{59}}$ and is used for $2 \mathrm{D}$ materials. $\underline{\underline{60}}$ It can even be used in hydrogenated amorphous silicon, a disordered material with very broad peaks, as we showed in a previous theory-experiment collaboration. $\underline{\underline{61}}$ This method has resolution on the $\mu \mathrm{m}$ scale, or even down to nanometers with tip-enhanced Raman spectroscopy. $\underline{62}$ Synchrotron-based IR microscopy has also been used for local mapping of strain and related structural changes. $\underline{\underline{63}} \underline{\underline{64}}$

In this paper, we analyze vibrations under compressive and tensile strain for cubic $\mathrm{CH}_{3} \mathrm{NH}_{3} \mathrm{PbI}_{3}$ and determine the calibration data needed to gauge local strain. The paper is organized as follows. In section II we detail our computational methods of structural opti- 
mization, calculation of normal mode frequencies and theoretical framework to understand frequency shifts in terms of perturbations of the dynamical matrix and mode eigenvectors. In section III, we discuss the behavior of phonon modes under uniaxial strain, understand the different behaviors in terms of structural changes and dynamical matrix analysis, find the best possible modes to probe local strain using Raman spectroscopy, and calculate the Grüneisen parameter and compare to other theory and experimental data. In section IV, we conclude and present the four best modes for measuring strain using Raman or IR spectra.

\section{Methods}

\subsection{Computational Details}

For structural optimization and phonon mode calculations we have used density functional theory (DFT) and density functional perturbation theory (DFPT) $\frac{65}{6}$ as implemented in Quantum ESPRESSO (version 6.1). $\underline{66} \underline{68}$ The Brillouin zone is sampled using a half-shifted $6 \times 6 \times 6$ Monkhorst-Pack grid with an energy cutoff of 100 Ry for the wave-functions. Atomic positions are optimized until the total force per atom is smaller than $1 \mathrm{meV} / \AA$; for the initial variable-cell relaxation, a 0.5 kbar stress convergence threshold is used. The Local Density Approximation $^{69}$ (LDA) with the Perdew-Wang (PW) parametrization ${ }^{70}$ is used for the exchange-correlation potential for all the calculations. Scalar relativistic Optimized NormConserving Vanderbilt (ONCV) pseudopotentials ${ }^{71}$ are used which treat $\mathrm{Pb} 5 d$ orbitals as valence. All the pseudopotentials are taken from Pseudō Dōjō̄ $\underline{\underline{2}, 73}$ (NC SR ONCVPSP v0.4) with standard accuracy. We have not considered spin-orbit coupling as it does not have much effect on interatomic interactions near equilibrium. $\underline{\underline{41}}$

The initial structure is taken from the work of Brivio et al.,,$\underline{41} \underline{\underline{4}}$ with the cation oriented close to the [100] direction, which was found to be slightly favored in molecular dynamics. It can be difficult to obtain an optimized exact cubic structure of MAPI without distortion

to other phases. $\stackrel{2}{2}$ Some experiments show the structure as cubic, $\underline{\underline{75}}$ others pseudo-cubic. $\underline{\underline{76}}, \underline{77}$ 
Experimental work reports the fast reorientation of the $\mathrm{CH}_{3} \mathrm{NH}_{3}^{+}$ion and also the rotation of $\mathrm{CH}_{3}$ and $\mathrm{NH}_{3}$ groups along the $\mathrm{C}-\mathrm{N}$ axis, and these rotations and reorientation are extremely fast $(\sim 14 \mathrm{ps}) \cdot \underline{\underline{78}}$ These motions can make a cubic symmetry averaged over space and time, but obviously cannot be captured in a static DFT calculation, which instead represents a single local snapshot. A periodic calculation imposes an artificial long-range order, but our dynamical matrix calculations suggest that this does not have an important effect on the vibrational properties (Sec. 3.3), consistent with the conclusion of Leguy et al. $\underline{\underline{49}}$ that dynamical disorder broadens peaks in MAPI but does not shift them significantly. Local asymmetry in the structure along with spin-orbit coupling has been calculated to cause splitting and an indirect gap. $\underline{79}, \underline{80} \mathrm{~A}$ theoretical study showed that changes in the $c / a$ ratio are coupled to the orientation of the $\mathrm{CH}_{3} \mathrm{NH}_{3}^{+}$ion. $\underline{\underline{23}}$ Our structural optimization makes the structure pseudo-cubic, in agreement with other calculations. $\underline{23}, \underline{41}$ As in standard DFT calculations, our lattice is at zero temperature, and the high temperature at which the cubic phase is observed does not enter into the calculations.

The equilibrium structural parameters are reported in Table 1. More detailed information, including results with different functionals, are reported in Table S1, along with bandgaps in Table S2 and the LDA bandstructure in Fig. S1 in agreement with previous work. $\underline{\underline{81}}$ Optimized crystallographic angles $\alpha, \beta$ and $\gamma$ are $90^{\circ}, 88.8^{\circ}$ and $90^{\circ}$ respectively. $\mathrm{Pb}-\mathrm{I}-\mathrm{Pb}$ angles are $164.65^{\circ}, 163.46^{\circ}$ and $173.92^{\circ}$ along $a, b$ and $c$ axes, respectively. The C-N bond-length is found to be $1.47 \AA$ with average $\mathrm{C}-\mathrm{H}$ and $\mathrm{N}-\mathrm{H}$ bond lengths of $1.1 \AA$ and $1.05 \AA$. Due to the pseudocubic lattice, off-centering of the $\mathrm{Pb}$ atom, cation orientation, and distortion of the $\mathrm{Pb}-\mathrm{I}$ cage, the structure has no symmetry, $\underline{\underline{36}}$ even when we checked the structure without the $\mathrm{CH}_{3} \mathrm{NH}_{3}^{+}$ion or with the $\mathrm{H}$ atoms removed. Our final relaxed cation orientation has the $\mathrm{C}-\mathrm{N}$ bond lying in the (010) plane, at an angle of $23.3^{\circ}$ to the $a$-axis ([100] direction).

Before applying strain to the structure, the convergence of the phonon frequencies with respect to the $k$-grid and phonon self-consistency threshold are checked, and all the calcula- 
Table 1: Optimized cell parameters for cubic $\mathrm{CH}_{3} \mathrm{NH}_{3} \mathrm{PbI}_{3}$ from DFT energy minimization and comparison with previous studies using DFT/PBEsol, $\underline{41,74}$ powder neutron diffraction (PND) ${ }^{75}$ and single crystal XRD ${ }^{77}$ methods.

\begin{tabular}{lcccccc}
\hline \hline & $\mathbf{a}(\AA)$ & $\mathbf{b}(\AA)$ & $\mathbf{c}(\AA)$ & $\alpha$ (deg.) & $\beta($ deg. $)$ & $\gamma($ deg. $)$ \\
\hline DFT/LDA & 6.163 & 6.115 & 6.267 & 90.00 & 88.80 & 90.00 \\
DFT/PBE & 6.499 & 6.410 & 6.532 & 90.00 & 88.66 & 90.00 \\
DFT/PBEsol & 6.291 & 6.248 & 6.378 & 90.00 & 88.64 & 90.00 \\
DFT/PBEsol $\underline{41.74}$ & 6.289 & 6.229 & 6.374 & 90.00 & 88.74 & 89.99 \\
PND (352 K) $)^{75}$ & 6.317 & 6.317 & 6.317 & 90.00 & 90.00 & 90.00 \\
XRD (400 K) $)^{\underline{77}}$ & 6.311 & 6.311 & 6.316 & 90.00 & 90.00 & 90.00 \\
\hline \hline
\end{tabular}

tions are done using phonon self-consistency threshold $10^{-16}$ to avoid imaginary frequencies at the $\Gamma$ point. We compared different functionals (PBE, $\underline{\underline{82}}$ PBEsol $\underline{83}$ and LDA $\underline{69}$ ) and found only small changes in mode frequencies at $q=0$. For low-frequency phonons, the pattern is $\mathrm{LDA}>\mathrm{PBEsol}>\mathrm{PBE}$ whereas for medium $\left(800-1600 \mathrm{~cm}^{-1}\right)$ and high $\left(2900-3200 \mathrm{~cm}^{-1}\right)$ frequencies it is $\mathrm{LDA}<\mathrm{PBEsol}<\mathrm{PBE}$ with most deviation at high frequencies, as shown in Fig. 1. For this comparison, we have performed the variable-cell relaxation using the same functional used to calculate the phonon frequencies.
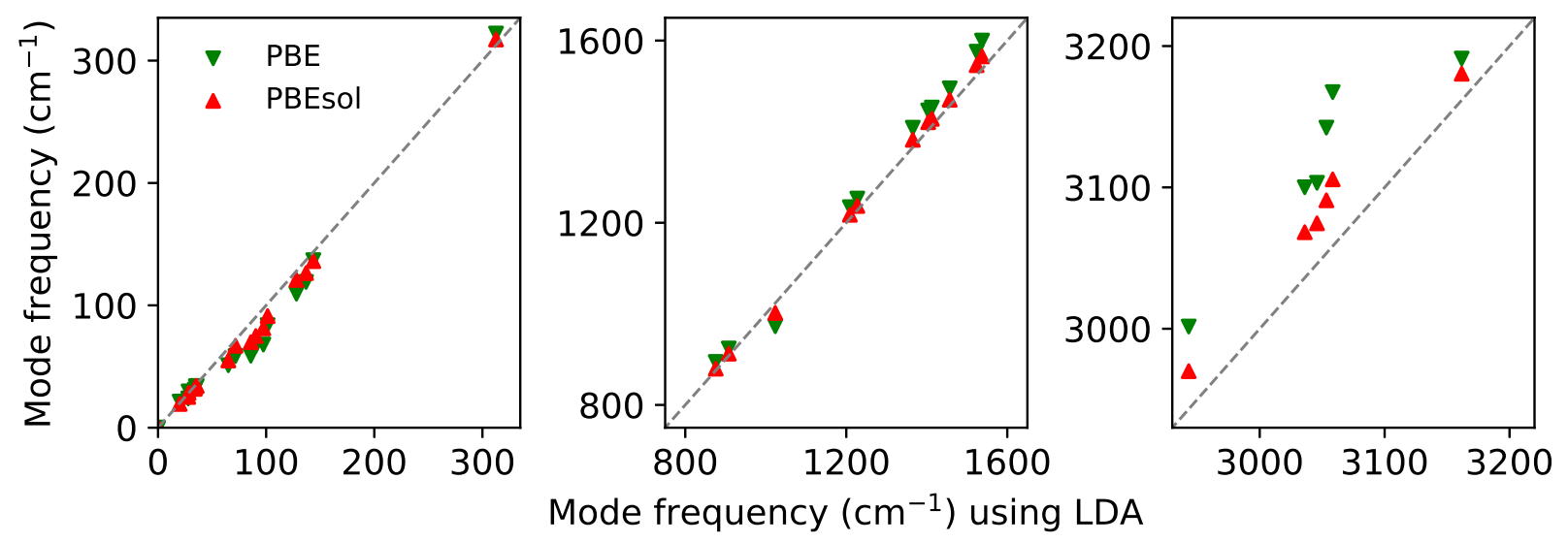

Figure 1: Comparison of $q=0$ phonon frequencies calculated with different functionals, showing good agreement across the low- and mid-frequency ranges, and some deviations at high frequencies. 
For reference, we performed similar calculations for the well studied case of c-Si (Fig. S2) and the result follows the same low frequency pattern LDA $>$ PBEsol $>$ PBE that we have found in cubic MAPI. We apply uniaxial strain in the [100] direction and plot frequency changes; the three degenerate optical phonons are split into a doublet and singlet. Results are shown in Table S3 and Fig. S2. LDA in fact has the best agreement with experiment $\underline{84}, 85$ for both frequency and slope, in this case; PBEsol is similar, but PBE is more different.

Moving now to the effect of strain on cubic MAPI, we applied up to 1\% uniaxial compressive (negative, in our convention) and tensile (positive) strain along the three crystallographic directions [100], [010] and [001] to the optimized zero-strain structure. Shear strains were not calculated as they generally have a smaller effect on the frequencies ${ }^{59}$, and are less commonly found in thin films. We considered results up to $\pm 0.4 \%$ strain where the changes in frequency with the applied strain are typically at least $1 \mathrm{~cm}^{-1}$, which is generally measurable in Raman spectroscopy. $\underline{\underline{45}}$ Strain more than $\pm 0.4 \%$ gives more signal-to-noise ratio, but more modes also become nonlinear. To make sure that this strain range is appropriate for studying linear responses, we have also computed the elastic constants by applying isotropic, tetragonal, and trigonal strain to the cubic MAPI lattice and thereby calculating elastic constants $C_{11}$, $C_{12}$ and $C_{44}$ following the procedure based on a quadratic fit of total energy. ${ }^{86}$ Within the range of $\pm 0.4 \%$, our result with LDA shows reasonable agreement with previously published results (Table 21). Note that we have separately calculated each diagonal element of the elastic constant tensor, and found some differences between them due to the pseudocubic lattice and lack of symmetry, whereas previous reports appeared to assume perfect cubic symmetry and calculated only $C_{11}$ and $C_{44} \cdot \underline{26,87}$

Having confirmed reasonable results for LDA on phonon frequencies and elastic constants, we are using LDA for all our strain and phonon calculations as Quantum ESPRESSO can only provide Raman intensities for LDA. $\stackrel{88}{ }$ The code's ASR=crystal setting is used to enforce the acoustic sum rule (ASR) and make the acoustic modes exactly zero. ${ }^{89}$ For each mode, we have calculated the uniaxial mode Grüneisen parameter for [100], [010] and [001] 
Table 2: Calculated values of elastic constants in GPa for cubic $\mathrm{CH}_{3} \mathrm{NH}_{3} \mathrm{PbI}_{3}$.

\begin{tabular}{rrrrrrr}
\hline \hline & $\mathrm{C}_{11}$ & $\mathrm{C}_{22}$ & $\mathrm{C}_{33}$ & $\mathbf{C}_{44}$ & $\mathrm{C}_{55}$ & $\mathbf{C}_{66}$ \\
\hline LDA (this work) & 38.1 & 40.5 & 38.7 & 2.8 & 2.5 & 3.7 \\
PBE $^{87}$ & 27.1 & - & - & 9.2 & - & - \\
PBEsol+vdW $^{26}$ & 35.4 & - & - & 6.1 & - & - \\
PBEsol $^{26}$ & 30.9 & - & - & 3.2 & - & - \\
\hline \hline
\end{tabular}

crystallographic directions using the slope of the frequency versus strain graph, with the formula $\gamma_{i}=-\left(1 / \omega_{i}\right) d \omega_{i} / d \epsilon$, where $\epsilon$ is the applied strain and $\omega_{i}$ is the frequency of mode $i . \underline{61}$ By taking the weighted average over all the modes, we have calculated the temperaturedependent Grüneisen parameter $\gamma=\Sigma_{i} \gamma_{i} C_{v, i} / \Sigma_{i} C_{v, i}$. This is connected to the macroscopic property of the material by the relation $\gamma=\alpha K_{T} / C_{v} \rho$ where $\alpha$ is volume thermal expansion coefficient, $K_{T}$ is isothermal bulk modulus, $C_{v}$ is heat capacity at constant volume and $\rho$ is the density. $\underline{\underline{90}}$ Raman intensities are calculated using the approach of Lazzeri et al. $\underline{\underline{88}}$ and IR intensities computed from Born effective charge tensors which are calculated as the variation of macroscopic polarization with the atomic displacement using the modern theory of polarization. $.65,91$

\subsection{Theoretical frame-work to understand the frequency change}

To obtain the normal mode frequencies, we solve the secular equation $\frac{65}{}$ given below:

$$
\sum_{J, \beta} D_{I \alpha, J \beta} u_{J \beta}=\omega^{2} u_{I \alpha}
$$

where $I, J$ denote atoms within the unit cell; $\alpha, \beta$ represent $x, y$ and $z$ directions; $D_{I \alpha, J \beta}$ is the dynamical matrix of the system; and $u_{I \alpha}$ represents the mode eigenvector. The dynamical 
matrix can be expressed as

$$
D_{I \alpha, J \beta}=\frac{1}{\sqrt{M_{I} M_{J}}}\left(\frac{\partial^{2} E}{\partial R_{I \alpha} \partial R_{J \beta}}\right)
$$

where $E$ is the total energy of the system, $R_{I}$ denotes the position vector of atom $I$, and $M_{I}$ denotes mass of atom $I$. For non-strained condition, the normal-mode frequency $\omega$ will be represented as $\omega_{0}$. To treat the variation of $\omega$ with strain, we write $\omega^{2}$ as

$$
\omega^{2}=\sum_{I \alpha, J \beta} u_{I \alpha}^{*} D_{I \alpha, J \beta} u_{J \beta}
$$

Strain can be treated as a perturbation to the vibrational properties of the material. The perturbation changes the dynamical matrix as well as the mode eigenvectors of the system, as given in equations (41) and (5) (up to quadratic order):

$$
\begin{gathered}
D_{I \alpha, J \beta}^{\epsilon}=D_{I \alpha, J \beta}^{0}+\sum_{l, m} \frac{\partial D_{I \alpha, J \beta}}{\partial \epsilon_{l m}} \epsilon_{l m}+\frac{1}{2} \sum_{l, m, l^{\prime}, m^{\prime}} \frac{\partial^{2} D_{I \alpha, J \beta}}{\partial \epsilon_{l m} \partial \epsilon_{l^{\prime} m^{\prime}}} \epsilon_{l m} \epsilon_{l^{\prime} m^{\prime}}+\cdots \\
u_{J \beta}^{\epsilon}=u_{J \beta}^{0}+\sum_{l, m} \frac{\partial u_{J \beta}}{\partial \epsilon_{l m}} \epsilon_{l m}+\frac{1}{2} \sum_{l, m, l^{\prime}, m^{\prime}} \frac{\partial^{2} u_{J \beta}}{\partial \epsilon_{l m} \partial \epsilon_{l^{\prime} m^{\prime}}} \epsilon_{l m} \epsilon_{l^{\prime} m^{\prime}}+\cdots
\end{gathered}
$$

Since the mode eigenvectors are real in our case at $q=0$, we can use $u_{I \alpha}^{*}=u_{I \alpha}$. If we apply the perturbed dynamical matrix with perturbed eigenvector to equation (3), we can calculate the change in frequency as

$$
\begin{array}{r}
\omega_{\epsilon}^{2}-\omega_{0}^{2}=\sum_{I \alpha, J \beta} \sum_{l, m}\left\{u_{I \alpha}^{0}\left(\frac{\partial D_{I \alpha, J \beta}}{\partial \epsilon_{l m}}\right) u_{J \beta}^{0}\right\} \epsilon_{l m}+ \\
\sum_{I \alpha, J \beta} \sum_{l, m, l^{\prime}, m^{\prime}}\left\{\frac{1}{2} u_{I \alpha}^{0}\left(\frac{\partial^{2} D_{I \alpha, J \beta}}{\partial \epsilon_{l m} \partial \epsilon_{l^{\prime} m^{\prime}}}\right) u_{J \beta}^{0}+\left(\frac{\partial u_{I \alpha}}{\partial \epsilon_{l m}}\right) D_{I \alpha, J \beta}^{0}\left(\frac{\partial u_{J \beta}}{\partial \epsilon_{l^{\prime} m^{\prime}}}\right)+\right. \\
\left.2 u_{I \alpha}^{0}\left(\frac{\partial D_{I \alpha, J \beta}}{\partial \epsilon_{l m}}\right)\left(\frac{\partial u_{J \beta}}{\partial \epsilon_{l^{\prime} m^{\prime}}}\right)+u_{I \alpha}^{0} D_{I \alpha, J \beta}^{0}\left(\frac{\partial^{2} u_{I \alpha}}{\partial \epsilon_{l m} \partial \epsilon_{l^{\prime} m^{\prime}}}\right)\right\} \epsilon_{l m} \epsilon_{l^{\prime} m^{\prime}}+\cdots
\end{array}
$$


The above equation can be used to find the change in frequency $\underline{59}$ due to strain $\epsilon$ as

$$
\Delta \omega \approx \frac{\omega_{\epsilon}^{2}-\omega_{0}^{2}}{2 \omega_{0}}
$$

For silicon, a simple matrix equation for the Raman-active transverse optical (TO) modes has been theoretically derived $\underline{54}, \underline{92}$ and experimentally verified, $\underline{\underline{92}}$ but for the pseudo-cubic perovskite the secular equation is more complicated as it has five different types of atoms and has no symmetry or degeneracy. Instead, to analyze this situation, we have considered changes in eigenvectors with strain, as well as the changes in the dynamical matrices to clearly understand which atomic interactions contribute significantly to the changes in phonon frequencies, as detailed in section 3.3.

\section{Results and Discussion}

\subsection{Behavior of phonon modes under uniaxial strain}

Calculated normal modes at $q=0$ of cubic $\mathrm{CH}_{3} \mathrm{NH}_{3} \mathrm{PbI}_{3}$ show three distinct frequency regions - low $\left(20-350 \mathrm{~cm}^{-1}\right)$, medium $\left(850-1600 \mathrm{~cm}^{-1}\right)$ and high $\left(2900-3200 \mathrm{~cm}^{-1}\right)$ - as described for cubic as well as orthorhombic and tetragonal structures in previous studies. $\underline{\underline{41}}$ Low-frequency modes are mainly due to the vibration of $\mathrm{PbI}_{6}$ octahedra and some coupling between the $\mathrm{PbI}_{6}$ octahedra and $\mathrm{CH}_{3} \mathrm{NH}_{3}^{+}$ion, while the medium and high frequency modes involve the vibration of the $\mathrm{CH}_{3} \mathrm{NH}_{3}^{+}$ion. Since pseudo-cubic $\mathrm{MAPbI}_{3}$ does not have any symmetry, $\underline{\underline{36}}$ it is not possible to assign spectral activity to any particular mode using group theory. All modes show both IR and Raman activity to some degree.

Our ab initio IR and Raman results are shown in Fig. 2, Calculated $\Gamma$-point phonon modes are convolved with $1 \mathrm{~cm}^{-1}$ Lorentzian broadening and compared with the calculations of F. Brivio et al.. $\stackrel{\underline{4}}{=}$ The difference in results are primarily due to our use of LDA and their use of PBEsol, as expected from Fig. 1. Calculated frequencies match well with published 


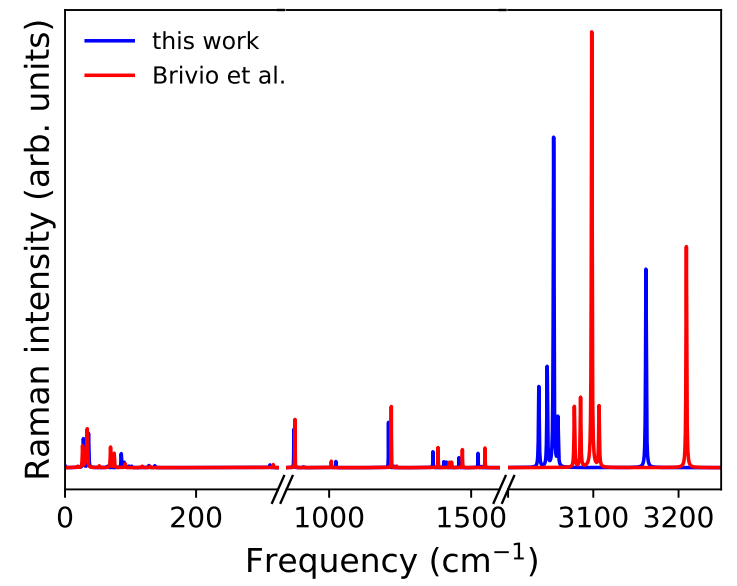

(a)

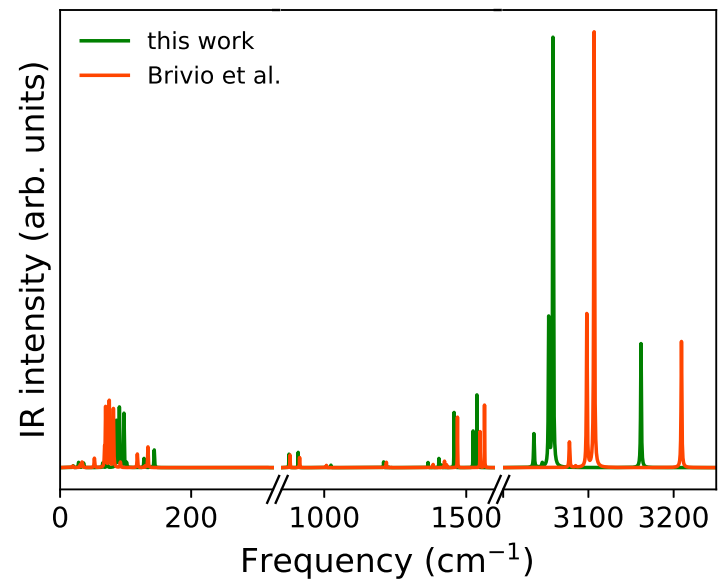

(b)
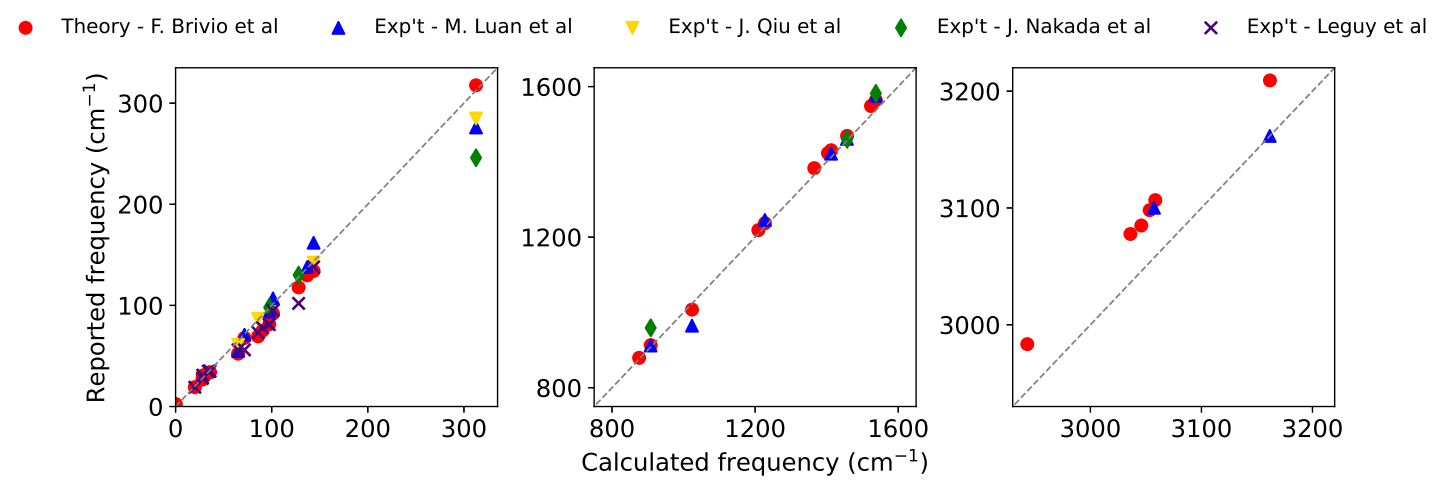

(c)

Figure 2: Comparison of calculated Raman and infrared frequencies. (a) Comparison of Raman spectrum convolved with $1 \mathrm{~cm}^{-1}$ Lorentzian broadening, with calculation of F. Brivio

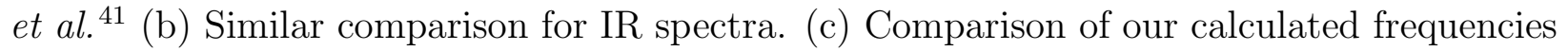
with published theoretical ${ }^{41}$ and experimental results: IR in Luan et al., $\underline{42}$ Raman in Qiu et al. $\underline{\underline{43}}$ and Nakada et al., $\underline{45}$ and $\mathrm{THz}$ in Leguy et al. $\underline{\underline{49}}$

theoretical results, $\stackrel{41}{\underline{4}}$ and experimental measurements from $\mathrm{THz}$ at low frequencies $\underline{49}$ and Raman $\frac{43,45}{4}$ and $\operatorname{IR}^{42}$ at medium to high frequencies.

We have 12 atoms in our unit cell for cubic $\mathrm{CH}_{3} \mathrm{NH}_{3} \mathrm{PbI}_{3}$, which gives 36 phonon modes, of which 3 have exactly zero frequency according to the ASR. For the other 33 modes we plotted frequency vs. applied strain (both compressive and tensile). Different patterns are noticed for different modes. Some of them are linear, some are parabolic, and some are neither (Fig. 4). For comparison, c-Si shows linear changes for small strain, and a splitting of degeneracy (Fig. S2). Given the much more complicated structure of the perovskite and 

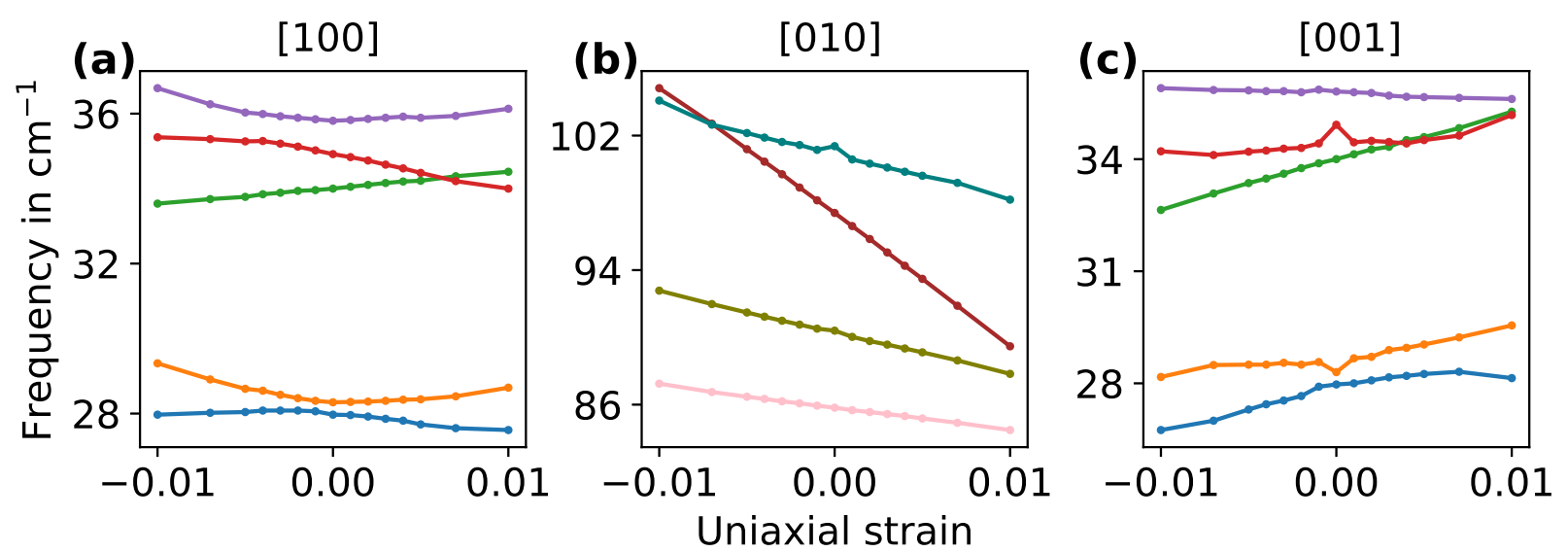

Figure 3: Frequencies of modes which approach or cross under strain, giving rise to parabolic or irregular behavior in frequency vs. strain.

lack of symmetry (hence no degeneracy), more complex behavior is observed. Low-frequency modes $\left(27.97 \mathrm{~cm}^{-1}, 28.3 \mathrm{~cm}^{-1}, 34.92 \mathrm{~cm}^{-1}\right.$ and $\left.35.81 \mathrm{~cm}^{-1}\right)$, which closely approach other modes under strain, show the parabolic pattern expected for an avoided crossing in phonons (or electronic bands), along with mixing and exchange of eigenvector character on either side of the crossing. The modes which approach or cross under strain are shown in Fig. 3 , Using the eigenvectors as a guide, in a few cases we found crossing of modes under strain, and therefore relabeled the modes to maintain a continuous character. Other modes with irregular behavior show even more mixing between modes, in a non-perturbative way, which we attribute to strong coupling to structural changes as discussed in Sec 3.2. Those modes where the frequency change is mostly linear can be categorized into four different categories: i) slopes of [100] and [010] strain are of same sign while slope of [001] is opposite sign (e.g. $\left.143.6 \mathrm{~cm}^{-1}, 3053.38 \mathrm{~cm}^{-1}\right)$, ii) slopes of [010] and [001] strain are of same sign while slope of [100] is of opposite sign (e.g. $\left.908.09 \mathrm{~cm}^{-1}, 1403.54 \mathrm{~cm}^{-1}\right)$, iii) slopes of [100] and [001] strain are of same sign while slope of [010] is of opposite sign $\left(e . g .20 \mathrm{~cm}^{-1}, 34 \mathrm{~cm}^{-1}\right)$ and iv) all slopes have same sign for [100], [010] and [001] strains (e.g. $\left.97.4 \mathrm{~cm}^{-1}, 312.59 \mathrm{~cm}^{-1}\right)$. The number of modes falling under different slope categories are given in Table S5 and the frequency change pattern for most of the modes is linear. There are some modes for which the slopes are almost the same for two different directions. For example, $97.4 \mathrm{~cm}^{-1}$ has $\mathrm{Pb}-$ 


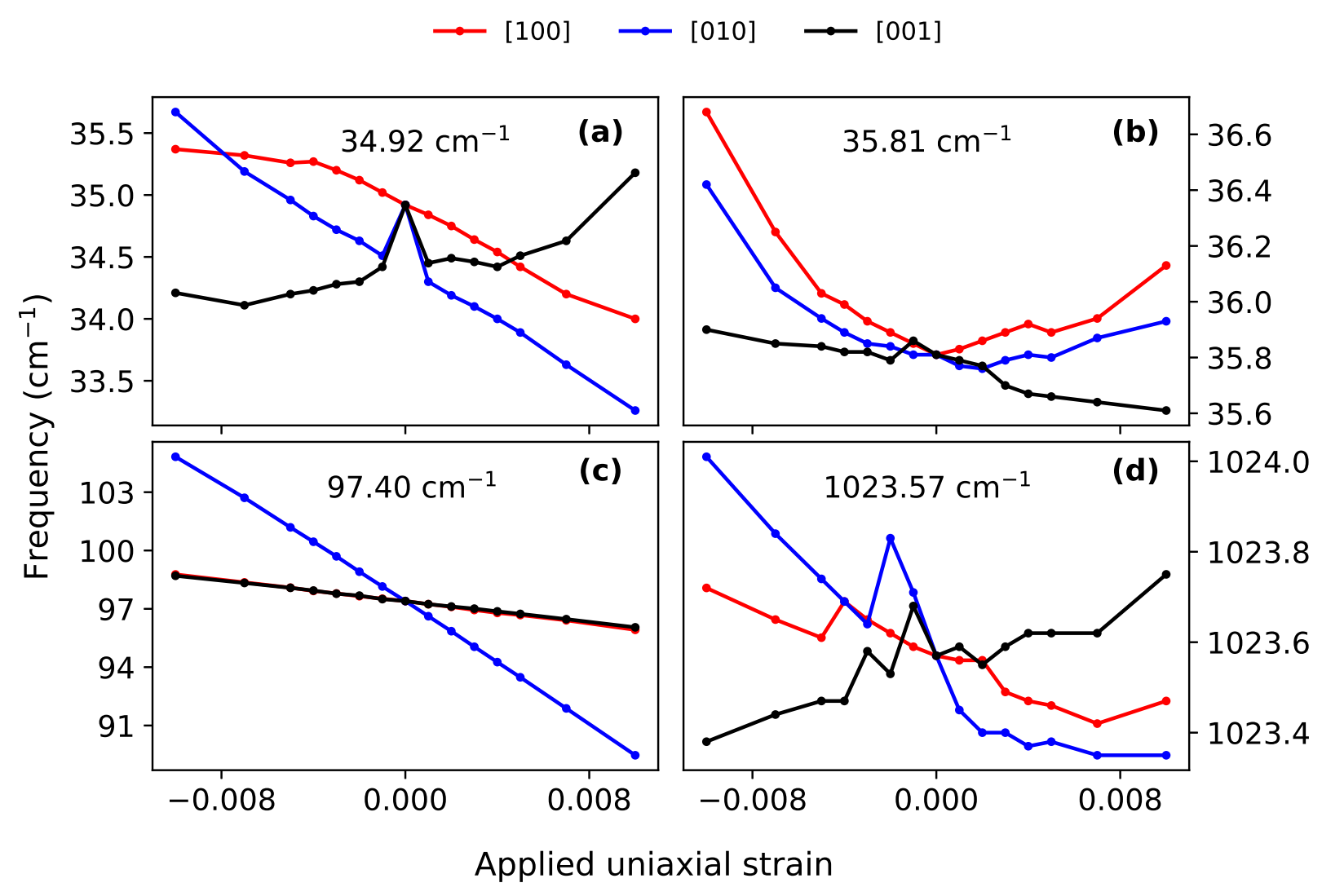

Figure 4: Typical patterns for frequency changes with applied compressive and tensile strain in three crystallographic directions [100], [010] and [001]. (a) Deep kink at zero strain for [010] and [001] but otherwise almost linear. (b) Parabolic pattern for [100] and [010] uniaxial strain. (c) Linear pattern. (d) Erratic pattern, neither linear nor parabolic.

I-Pb bending perpendicular to both [100] and [001], giving rise to the same slopes for these strain directions. For $1365.3 \mathrm{~cm}^{-1}$, symmetric umbrella type $\mathrm{C}-\mathrm{H}$ bending has components in all directions and has comparable behavior for [100] and [010] strain directions. These behaviors indicate approximate symmetries of particular modes despite the lack of overall symmetry. In Fig. 4, we provide four representative modes' frequency vs. strain patterns.

To understand these behaviors, we plotted eigenvectors (displacement patterns) for each mode, contributed by each atom in the unit cell as $\left|u_{I}\right|=\sqrt{\left(u_{I x}^{2}+u_{I y}^{2}+u_{I z}^{2}\right)}$; those for the chosen four representative modes are given in Fig. 5. We noticed that the change in frequency with strain is linear when the eigenvector does not change appreciably but the dynamical matrix does, as the effect of $2^{\text {nd }}$ order change in dynamical matrix comes in 


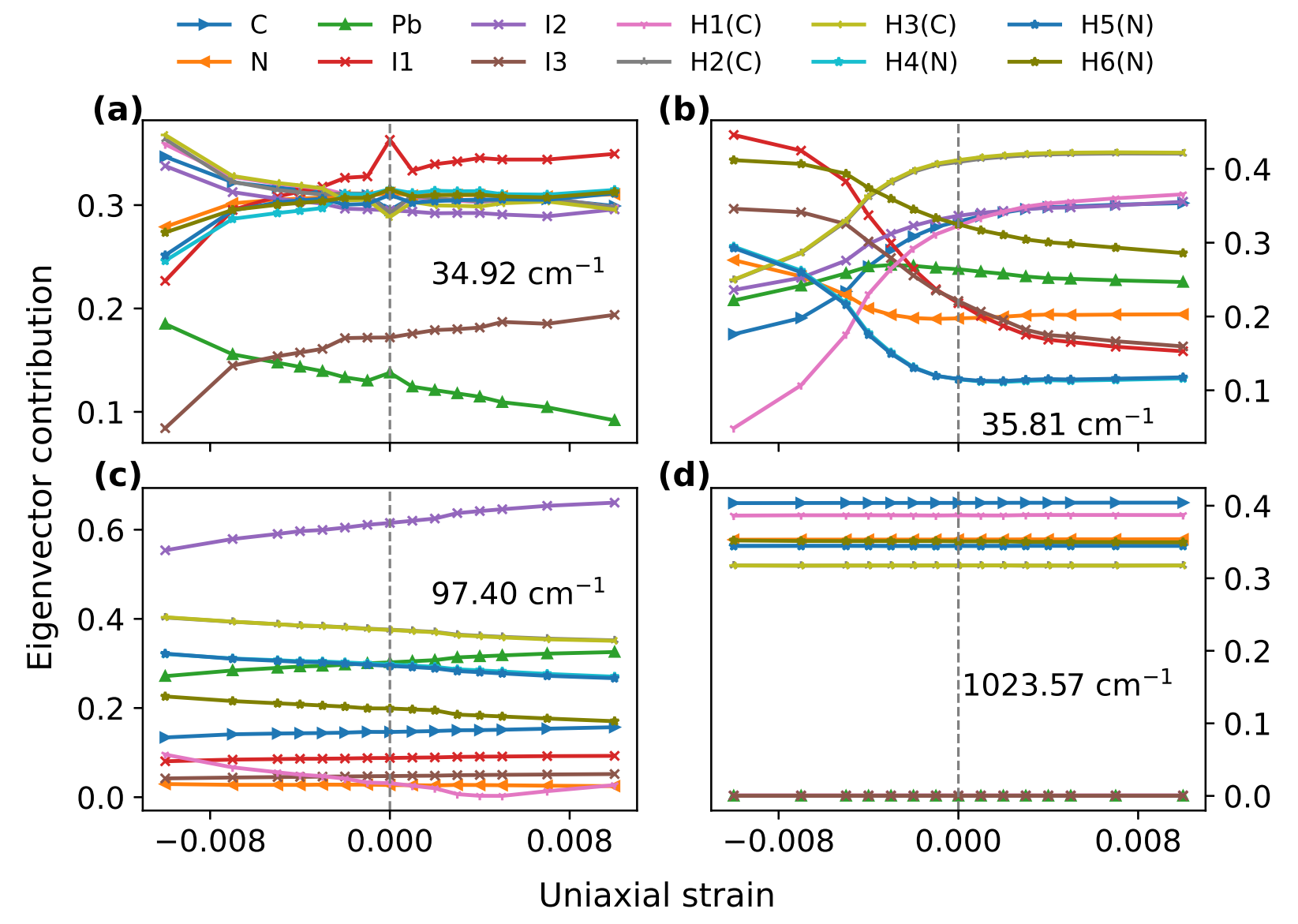

Figure 5: Contributions of each atom in the eigenvectors for each of the representative modes presented in Fig. 4. Uniaxial strains are in the (a) [010], (b) [100], (c) [001], and (d) [001] directions, showing connections between parabolic or other nonlinear frequency behavior in (a) and (b) with significant changes in mode character, while (c) has little character change and linear frequency behavior, and (d) anomalously has little character change but erratic frequency behavior which will be explained in 3.2 .

only at $2^{\text {nd }}$ order (Eqn. 6). Due to the lack of symmetry, the dynamical matrix elements always have a change with strain in this system. On the other hand, if the eigenvector does change, we can have some non-linear effect in the frequency change which can be understood from the second-order part of Eqn. 6. In most cases, the dynamical matrix change is large enough to produce effects beyond quadratic. For example, Fig. 4(a) shows a kink at zero for the frequency for both [010] and [001] strain while for [100] strain it is almost linear (parabolic under compressive strain). We can see that the change in mode eigenvector for the corresponding mode shows a drastic change at zero for strain along [010] direction (Fig. 
5(a)) and the mode's character is a combination of translation of the $\mathrm{CH}_{3} \mathrm{NH}_{3}^{+}$ion along [001] direction and Pb-I-Pb rocking mode. Fig. 4(b) shows parabolic frequency changes for [100] and [010] strain which can be understood from the changes of the eigenvector and close approach with another mode (Fig. 5(b)). This mode is a combination of $\mathrm{CH}_{3} \mathrm{NH}_{3}^{+}$ ion libration in the [010] direction and a Pb-I-Pb rocking mode. In Fig. $4(\mathrm{c})$ we can see that the frequency change is linear and if we check the corresponding change in eigenvector (Fig. [5(c)) we can see that there is very little change with strain which as per Eqn. 6 will produce a linear pattern. The mode character for this mode is libration and spin (all the $\mathrm{H}$ atoms attached to $\mathrm{C}$ and $\mathrm{N}$ are rotating in the same direction) of $\mathrm{CH}_{3} \mathrm{NH}_{3}^{+}$ion, and $\mathrm{Pb}-\mathrm{I}-$ $\mathrm{Pb}$ bending. Finally, in Fig. $4(\mathrm{~d})$ we found that the frequency change pattern is irregular despite very little change in atomic contributions to the eigenvector (Fig. [5(d)). This is because the $x, y$ and $z$ components for $\mathrm{C}, \mathrm{N}$ and $\mathrm{H}$ are changing irregularly with strain even though the sum of squares over the Cartesian directions is constant for each atom. This is a pure molecular mode with symmetric $\mathrm{C}-\mathrm{H}$ and $\mathrm{N}-\mathrm{H}$ bending with $\mathrm{C}-\mathrm{N}$ stretch. We find that generally $\mathrm{C}-\mathrm{H}$ and $\mathrm{N}-\mathrm{H}$ vibrations are associated with nonlinear changes in the mode character under strain. We will see by structural and dynamical analysis that C-H, $\mathrm{N}-\mathrm{H}$ vibrations are important for most of the frequency change under strain. Corresponding plots for all modes are given in Fig. S12.

\subsection{Structural Changes under uniaxial strain}

While highly symmetric structures such as c-Si do not have internal parameters that can change with strain, in hybrid perovskites, the structure evolves under applied strain, which can play a role in vibrational changes. The significant changes are a sign of the anharmonicity of MAPI. Lengths of bonds parallel to strain are changed most, but those perpendicular to the direction of strain are also affected. For example, the Pb-I bond length along [100] changes most for strain along [100] but Pb-I bond lengths along other two perpendicular directions, such as along [010] and [001] are also changing as shown in Fig. 6(a). This 


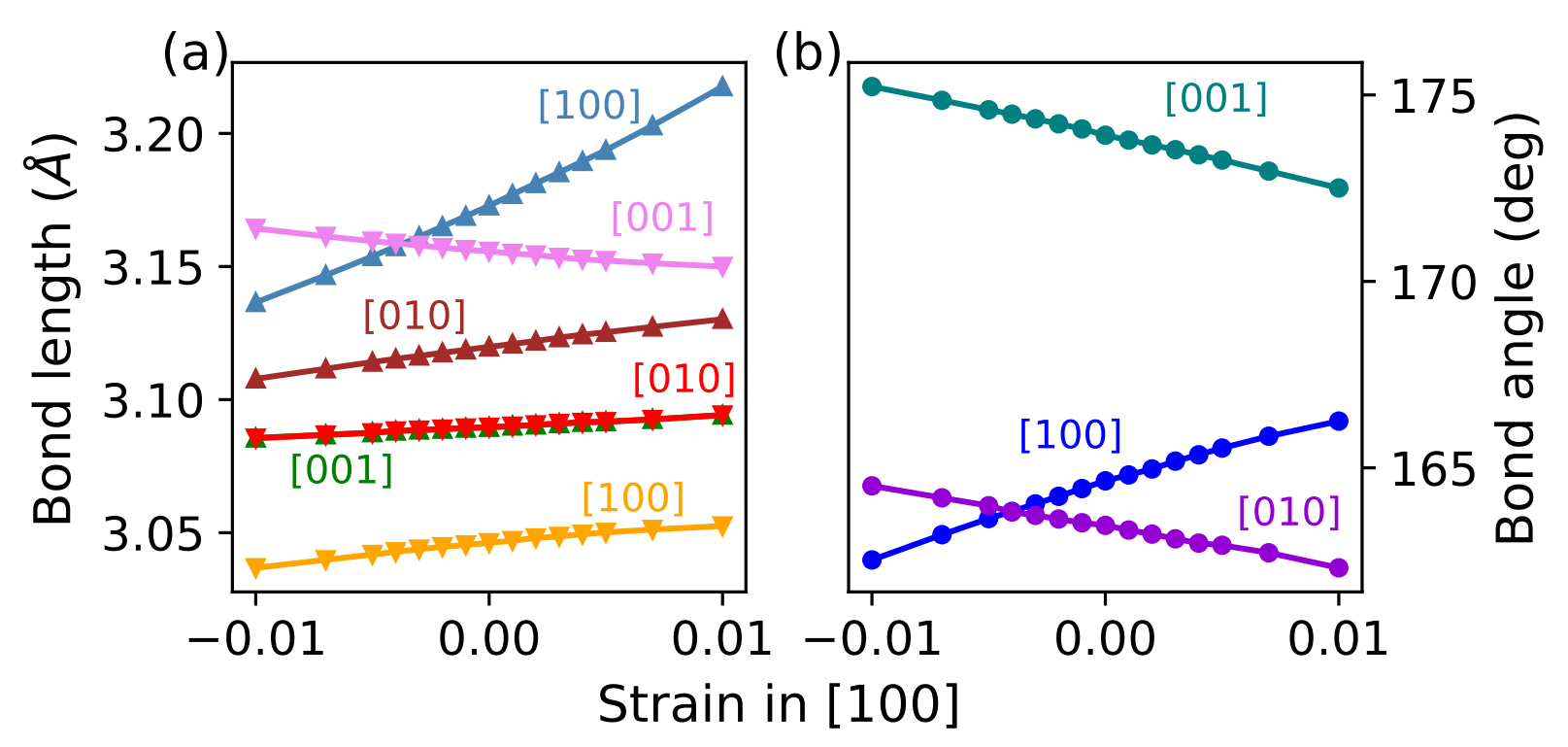

Figure 6: Change in $\mathrm{Pb}-\mathrm{I}$ bond length and $\mathrm{Pb}-\mathrm{I}-\mathrm{Pb}$ bond angle in cubic $\mathrm{CH}_{3} \mathrm{NH}_{3} \mathrm{PbI}_{3}$ for uniaxial [100] strain, showing fairly linear relationships, and a buckling of the Pb-I cage. These are the six $\mathrm{Pb}-\mathrm{I}$ bonds, labeled by the crystal direction of the bond, and three $\mathrm{Pb}-\mathrm{I}-$ $\mathrm{Pb}$ angles along [100], [010] and [001] directions respectively.

change in bond lengths in the perpendicular direction to the strain is more prominent when the strain is acting perpendicular to the direction of the methylammonium ion which is close to [100] (Fig. S3). The Pb-I-Pb bond angles also change with applied strain: they decrease with compressive strain acting parallel to the bond angle and increase when it is acting perpendicular to the bond angle as can be seen in Fig. 6(b). Pb-I-Pb bond angle which is along [100] decrease, and those along [010] and [001] directions increase, for compressive strain along the [100] direction. Similar behavior is found in a theoretical study for tetragonal structure where the $\mathrm{Pb}-\mathrm{I}-\mathrm{Pb}$ bond angle which is parallel to the strain direction increases for tensile strain and decreases for compressive strain, but the $\mathrm{Pb}-\mathrm{I}-\mathrm{Pb}$ bond angle perpendicular to the strain direction increases for compressive strain and decreases for tensile strain..$^{27}$ There are almost no changes $(<0.007 \%)$ in the $\mathrm{C}-\mathrm{N}$ bond length but the $\mathrm{N}-\mathrm{H}$ bond length (along [001]) decreases with compressive strain (Fig. S9). This reduction in N-H bond length supports the idea that compressive strain may be useful to stabilize the material. $\underline{.93,94}$

The $\mathrm{CH}_{3} \mathrm{NH}_{3}^{+}$ion, which lies in the $(010)$ or $x z$-plane also rotates with an increasing 
angle with respect to the [100] direction or $a$-axis under compressive strain and a decreasing angle under tensile strain (Fig. S11), with changes of up to $1.5^{\circ}$ over our strain range which is related to the change in $c / a$ ratio $^{23}$ as mentioned in Sec. 2.1. Rotation is largest for [010] strain. This may be due to the fact that the $\mathrm{CH}_{3} \mathrm{NH}_{3}^{+}$ion lies in the (010) plane perpendicular to [010]. Rotation of $\mathrm{CH}_{3} \mathrm{NH}_{3}^{+}$is also reported for tetragonal structure under compressive strain. ${ }^{27}$ The distances of $\mathrm{C}$ and $\mathrm{N}$ with their nearest $\mathrm{Pb}$ and $\mathrm{I}$ change with strains and the discontinuity in the $\mathrm{I}-\mathrm{N}, \mathrm{I}-\mathrm{C}, \mathrm{N}-\mathrm{Pb}$ and $\mathrm{C}-\mathrm{Pb}$ distances gives an indication why we see certain kinks or irregularities in the Raman shift vs strain graphs. For example, mode $128.01 \mathrm{~cm}^{-1}$ shows kinks in the frequency change pattern (Fig. S12) where C-H and $\mathrm{N}-\mathrm{H}$ are showing asymmetric bending modes with libration of the MA ion within the Pb-I cage, which is affecting the $\mathrm{C}-\mathrm{Pb}$ and I-N distances most. Similarly, for $1023.57 \mathrm{~cm}^{-1}$, we see that the irregularity is greater for [010] and [001] strain than [100]. We have further analyzed these changes in terms of the dynamical matrix in the next section. Full plots of structural parameters with each direction of strain are in Fig. S3-S11, and all atomic coordinates and phonon eigenvectors of relaxed strained structures are provided in the Supporting Information.

\subsection{Change in dynamical matrix due to uniaxial strain.}

To understand the different behaviors of the phonon modes under strain, we have analysed the dynamical matrix of each strained structure according to Section 2.2. We have calculated the changes in dynamical matrices and plotted the results as heat-maps in Fig. 7(a) and 7(c) to understand which changes in interatomic interactions are most significant for the vibrational frequency changes under strain and how they relate to the different linear/parabolic/irregular pattern observed. Fig. 7(b) shows the dynamical matrix for the unstrained lattice. Note that these plots are symmetric about the diagonal and the scales are different for the strained and non-strained cases. The largest elements are in the C, N, H block due to lighter masses (see Eqn. 2). Diagonal elements are generally larger than 


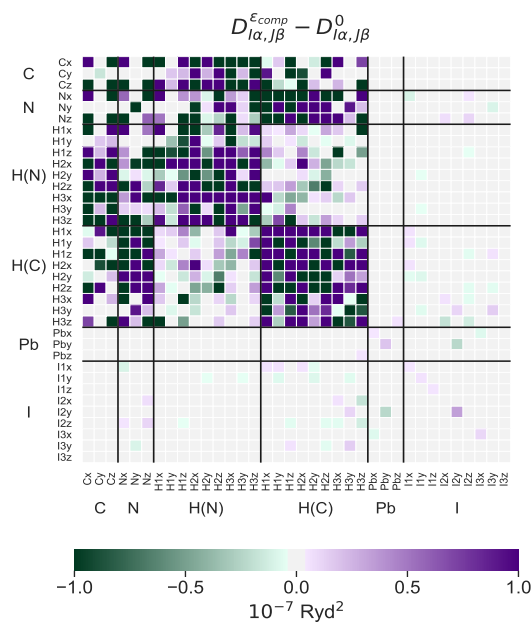

(a)

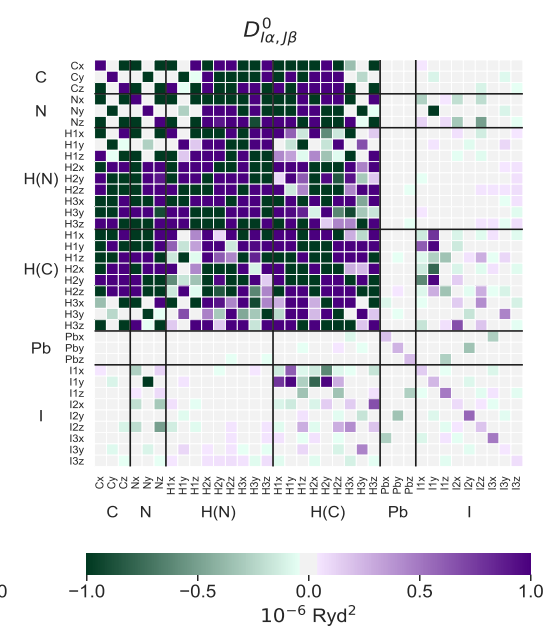

(b)

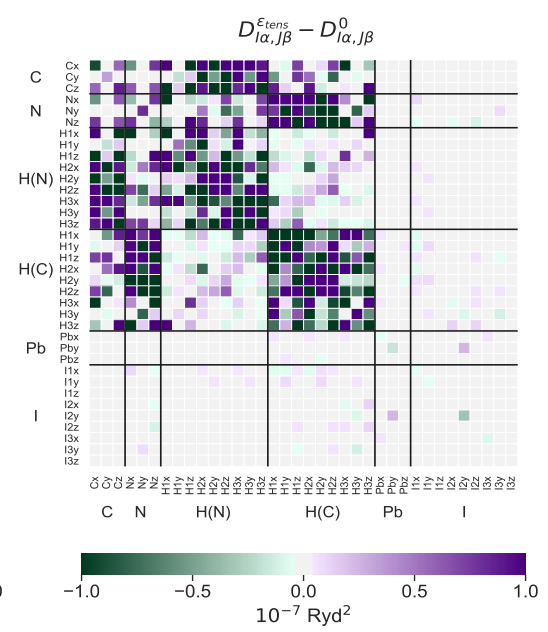

(c)

Figure 7: Dynamical matrix for strain along [010] direction. (a) Change in dynamical matrix for compressive strain $(\epsilon=-0.004)$. (b) Dynamical matrix at zero strain. (c) Change in dynamical matrix for tensile strain $(\epsilon=0.004)$. Symbols in both the axes represent atoms and their coordinates. For example, within $\mathrm{H}(\mathrm{N})$ block, $\mathrm{H} 3 \mathrm{z}$ denotes the $z$ coordinate of the third $\mathrm{H}$ attached to $\mathrm{N}$.

off-diagonal elements, as can be seen at Fig. 7(b). It can be also seen that changes due to compressive strain are more than those of tensile strain, which can be understood based on the typical curve of energy vs. bond length, e.g. the Morse potential. Since $\mathrm{Pb}$ and I are heavier than $\mathrm{C}, \mathrm{N}$ and $\mathrm{H}, \mathrm{Pb}-\mathrm{I}$ interactions will be significant most for low frequency modes. It can be noticed that there are very small interactions between $\mathrm{H}$ atoms and their nearest $\mathrm{Pb}$ or I atoms (Fig. 7(b)) which gives an indication that van der Waals interactions are of minor importance for vibrations of the cubic structure which is also supported by a previous study. $\frac{95}{}$ We have calculated also the dynamical matrix for a $2 \times 2 \times 2$ supercell, and found that the matrix elements are significantly smaller when the two atoms are in different primitive cells. This indicates that the artificial long-range order in our periodic structure does not make much difference in the vibrational properties compared to the true dynamical disorder.

From the change in dynamical matrix in Fig. 7(a) and Fig. 7(c), we can see that the component of $\mathrm{Pb}-\mathrm{I}$ interactions changes most when it is parallel to the direction of strain. 
For example, the $\mathrm{Pb}_{y}-\mathrm{I}_{y}$ interaction changes most for [010] strain whereas $\mathrm{Pb}_{x}-\mathrm{I}_{x}$ and $\mathrm{Pb}_{z}-\mathrm{I}_{z}$ interactions change most for [100] and [001] strains respectively (Fig. S14-S15). We have already seen in the structural changes that $\mathrm{Pb}-\mathrm{I}$ bond length is affected most when the stretches are along the direction of polarization of the bond. It is clear from Fig. 7(a) and Fig. 7(c) that Pb-I modes are significantly affected due to strain. It can also be seen that I-N coupling (lower left and upper right) is important and greater than I-C coupling which is due to the electrostatic interaction between the MA ion and the cage. These interactions play a significant role in the frequency shifts and are important for the medium and high frequency regions.

Given the success of analyzing vibration separately $\underline{\underline{47}}$ (lattice and ion), one might think that the lattice strain will not affect the MA ion, but this turns out not to be true. Among the hydrogens attached to $\mathrm{C}$, or the hydrogens attached to $\mathrm{N}$, there are significant changes with strain in the interactions, which explain the behaviors of some of the high frequency modes. Modes that have a large component of $\mathrm{C}-\mathrm{H}, \mathrm{N}-\mathrm{H}$ stretch have higher slopes in the high frequency region, whereas in the low frequency region large $\mathrm{Pb}-\mathrm{I}$ stretch or bend with C-H, N-H stretch is associated with large slope. C-H, N-H asymmetric stretch or $\mathrm{CH}_{3} \mathrm{NH}_{3}$ spin and torsion are found to have high slopes. It is found that slope values are high in the low and high frequency region and small in the medium frequency region. It is also found that the slope values are typically higher when the strain is perpendicular to the direction of the MA ion (along [010] and [001]) and lower when its along the direction of the MA ion i.e. along [100]. We attribute this asymmetry to the orientation of the C-H bonds: two of the $\mathrm{C}-\mathrm{H}$ and $\mathrm{N}-\mathrm{H}$ bonds lie closer to [010] than to [100], giving greater effects from [010]; one $\mathrm{C}-\mathrm{H}$ and one N-H bond lie parallel to [001], and these bond lengths are affected most by [010], and also show the greatest effects of any C-H or N-H bonds in any strain direction (Fig. S9, S10). Therefore the strain effects on the MA ion, and its interactions with the cage, are greatest when we apply strain along [010] and [001].

IR and Raman intensities are much higher in the high frequency region than in the low 
or mid-frequency regions, and are mainly contributed by $\mathrm{C}-\mathrm{H}$ and $\mathrm{N}-\mathrm{H}$ stretches. From our full phonon analysis, shown in Table S4, we found that low-frequency modes are mainly due to $\mathrm{Pb}-\mathrm{I}-\mathrm{Pb}$ vibration with translation or libration mode of the MA ion. The mid-frequency modes mainly consist of C-H and N-H asymmetric bending with some spin or twist. The high-frequency modes are mainly symmetric and asymmetric stretch of $\mathrm{C}-\mathrm{H}$ and $\mathrm{N}-\mathrm{H}$ bonds of the methylammonium ion. For all the modes, IR intensity vectors are lying in the plane, either in $x z(100)$ or in $x y$ (001) plane. Modes which have high slopes in all three directions - among them, high frequency modes $\left(3053.38 \mathrm{~cm}^{-1}, 3058.42 \mathrm{~cm}^{-1}, 3161.71 \mathrm{~cm}^{-1}\right)$ - are both highly IR- and Raman-active while low frequency modes $\left(27.97 \mathrm{~cm}^{-1}, 65.02 \mathrm{~cm}^{-1}\right.$, $85.82 \mathrm{~cm}^{-1}$ ) are less IR- and Raman-active. Modes involving libration and translation of the $\mathrm{CH}_{3} \mathrm{NH}_{3}^{+}$ion and $\mathrm{Pb}-\mathrm{I}-\mathrm{Pb}$ bending have high slopes along [100] direction while modes having high slope in [010] show $\mathrm{C}-\mathrm{H}$ and $\mathrm{N}-\mathrm{H}$ stretch in $\mathrm{CH}_{3} \mathrm{NH}_{3}^{+}$ion.

\subsection{Best modes to probe local strain in cubic $\mathrm{CH}_{3} \mathrm{NH}_{3} \mathrm{PbI}_{3}$}

An appropriate mode for strain mapping with IR/Raman microscopy should have i) a large IR or Raman intensity, for ease of detection; ii) a linear frequency change with strain, for simple and unique relation of frequency to strain; iii) a large slope for frequency vs. strain, for sufficient signal-to-noise ratio in measuring frequency shifts; and finally, iv) a small change in frequency with respect to cation orientation, ensuring the validity of our results in the presence of cation rotations at elevated temperature. $\underline{36}$

A difficulty in checking these frequency changes with cation orientation is that phonon calculations are not very meaningful except from a relaxed structure, and only a few cation orientations are stable. $\underline{41,79}$ We took our zero-strain structure and rotated the cation close to the [111] direction, and relaxed the structure. The result is $0.01 \mathrm{eV}$ higher in energy, and has the cation in the $x z$ plane, with the $\mathrm{C}-\mathrm{N}$ bond at an angle of $113.9^{\circ}$ (compared to $23.3^{\circ}$ for our main data set) with respect to [100] direction. From a phonon calculation, we find some mode frequency changes due to the $\mathrm{CH}_{3} \mathrm{NH}_{3}^{+}$rotation, typically by a few wavenumbers. 


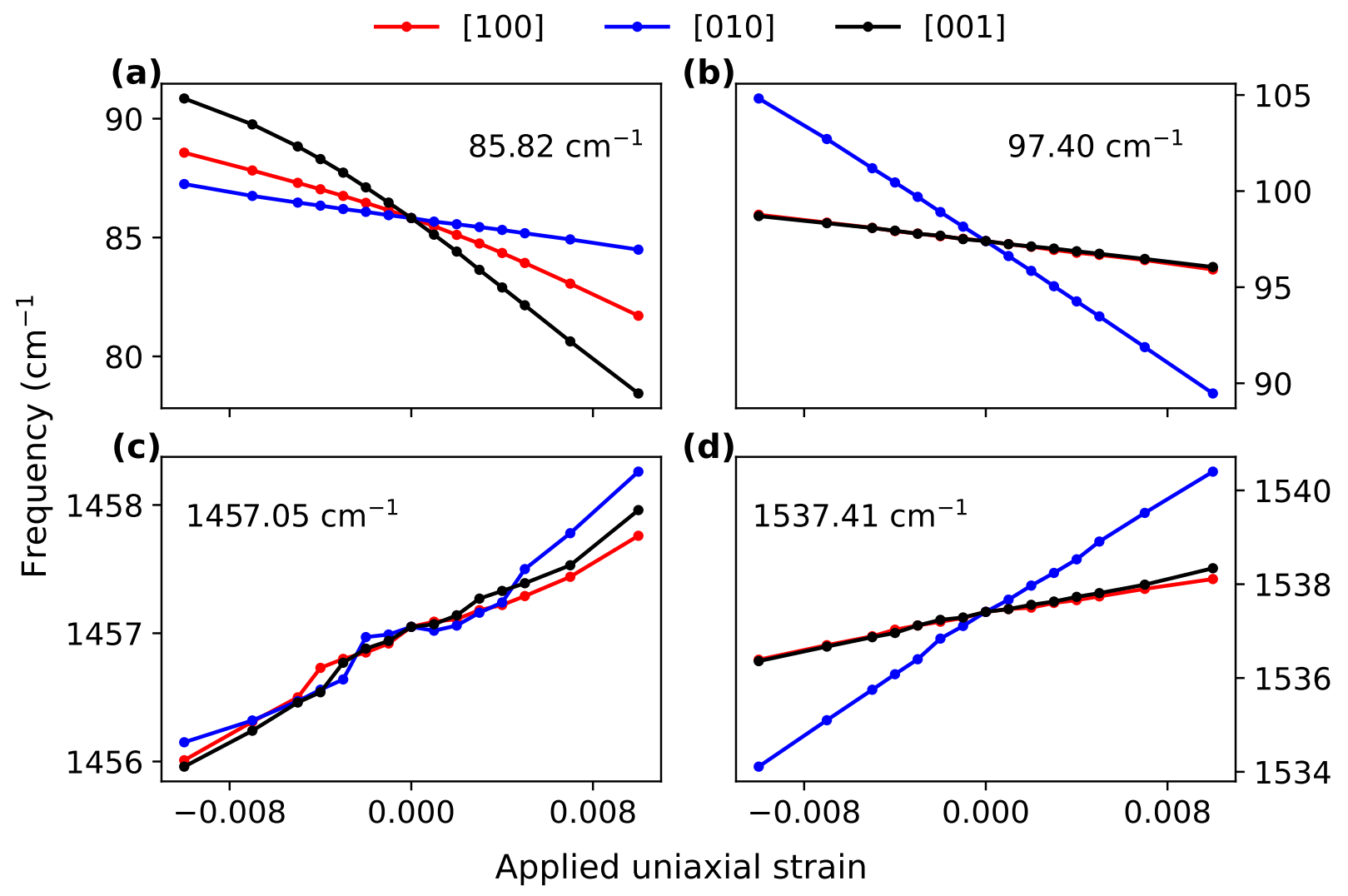

Figure 8: Frequency vs. strain for the best modes for IR/Raman microscopy to probe local strain, showing linear changes for robust calibration.

We may expect this variation to be a contribution to heterogeneous broadening of Raman peaks as ions rotate thermally. Indeed Nakada et al. report larger peak widths for high- and low-frequency modes than for mid-frequency modes, for all 3 crystal phases, $\underline{\underline{45}}$ in accordance with our results for frequency changes with ion rotation. The high- and low-frequency modes change most while the mid-frequency modes change less due to the $\mathrm{CH}_{3} \mathrm{NH}_{3}^{+}$rotation. This is because the mid-frequency region is mainly symmetric/asymmetric bending modes of the MA ion which are not affected much by rotation of the MA ion, whereas stretching modes at high and low frequency are the ones that change more when the distances change between the MA ion and the $\mathrm{Pb}-\mathrm{I}$ cage. For this reason, the same modes that have large frequency changes with ion rotation have large slopes with strain. Full results are shown in Table S4. If the mode frequency changes by a significant amount due to rotation of the molecule, our 


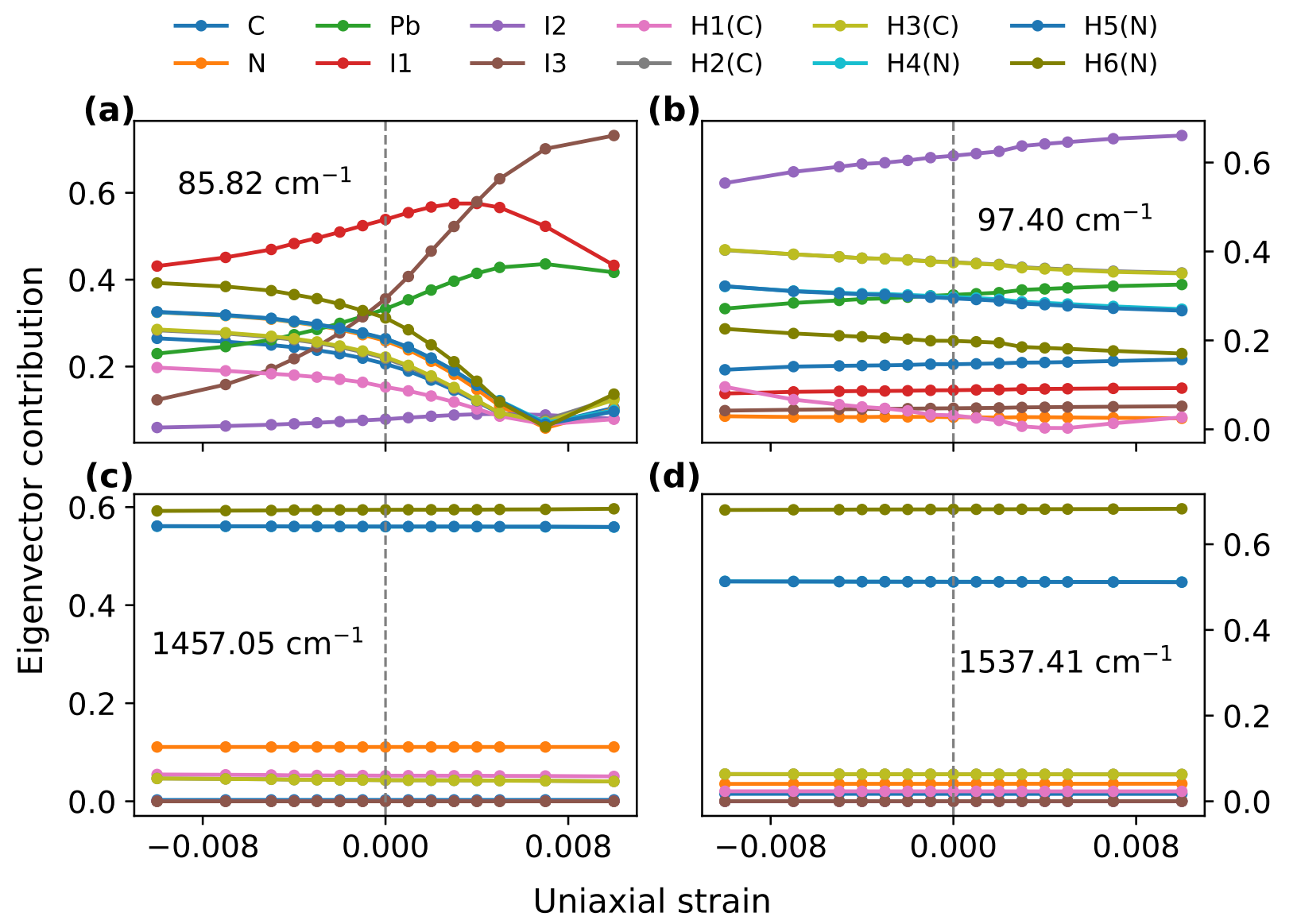

Figure 9: Mode eigenvector vs. strain for the best modes for IR/Raman microscopy to probe local strain, showing little change in mode character (except for (a)), associated with robust linear changes vs. strain.

static result may have a larger error, and the predicted frequency shifts due to strain will be less reliable. Therefore, we need to focus on those modes for which frequency change due to rotation of the MA ion is minimal.

After this analysis, we can identify the best modes for IR or Raman microscopy for probing local strain. $\underline{59,61}$ We note 4 suitable modes, whose properties are detailed in Table 3 and whose displacement patterns are shown in Fig. S13. A combination of all 4 modes can be used together for better precision, or even to find a best fit to 3 directions of uniaxial strain. Two are low-frequency $\mathrm{Pb}-\mathrm{I}$ modes and two are mid-frequency molecular modes. The frequency changes vs. strain are shown in Fig. 8 and the eigenvectors vs. strain are shown in Fig. 9, exhibiting little change in mode character and a robust linear frequency 
change. Each of these modes has all slopes positive or all slopes negative. In the case of triaxial strain or when cation rotations wash out directional dependence, the average of the three uniaxial slopes would be the appropriate slope according to Eq. 6. Having all uniaxial slopes with the same sign is convenient, because the three directions will reinforce each other rather than cancelling out, as could happen when the slopes have different signs. The IR and Raman intensity for these 4 modes show only moderate changes with strain (Fig. S16-17) as for c-Si optical modes,,$\underline{59}$ which we expect would not cause any problem for experimental measurement. We find another mode at $1365.3 \mathrm{~cm}^{-1}$ that has favorable properties with reasonable Raman intensity to probe local strain (Fig. S12); however it is not observed in experiment. The reason may be limitations of our model. Our structure is pseudocubic rather than cubic, and our calculation is static and done at $0 \mathrm{~K}$ at which this is not the stable phase. As a result, this mode may in fact lose its Raman activity due to dynamic average symmetry in the real high-temperature structure.

There is a final point to consider in assessing experimental feasibility. To be able to measure strain by Raman shifts, we need to obtain a frequency shift that is higher than the experimental resolution. For typical strain $\frac{26,27}{2}$ of $1 \%$ we can expect to obtain shifts -3.8 $\mathrm{cm}^{-1},-3.5 \mathrm{~cm}^{-1}, 0.75 \mathrm{~cm}^{-1}$ and $1.57 \mathrm{~cm}^{-1}$ for the favorable Raman modes at $85.8 \mathrm{~cm}^{-1}, 97.4$ $\mathrm{cm}^{-1}, 1457.1 \mathrm{~cm}^{-1}$ and $1537.4 \mathrm{~cm}^{-1}$, respectively based on the average slopes in Table 3 , Experimentally measured full widths at half maximum (FWHMs) for Raman spectra $\underline{42}, \underline{43}, 45$ around the low-frequency modes are $8 \mathrm{~cm}^{-1}, 10 \mathrm{~cm}^{-1}$, and $18 \mathrm{~cm}^{-1}$, and for the mode at $1460 \mathrm{~cm}^{-1}$, the FWHM is around $35 \mathrm{~cm}^{-1}$. One contribution to FWHM can be strain itself - inhomogeneous strain distributions within the Raman focus will result in broadening. $\underline{\underline{96}}$ Although these FWHMs are high, peak shifts can be resolved less than $1 \mathrm{~cm}^{-1}$, as seen for the temperature-dependent Raman shifts measured by Nakada et al. $\underline{\underline{45}}$ and in Strubbe et $a l . \underline{\underline{61}}$ on a-Si:H, because fitting of Gaussian peaks allows determination of differences in peak centers to much greater precision than the FWHM. Temperature dependent frequency shifts were measured for MAPI by Nakada et al. $\underline{\underline{45}}$ and they were able to measure shifts less than 1 
Table 3: Best modes for IR or Raman microscopy to probe local strain. ${ }^{a}$

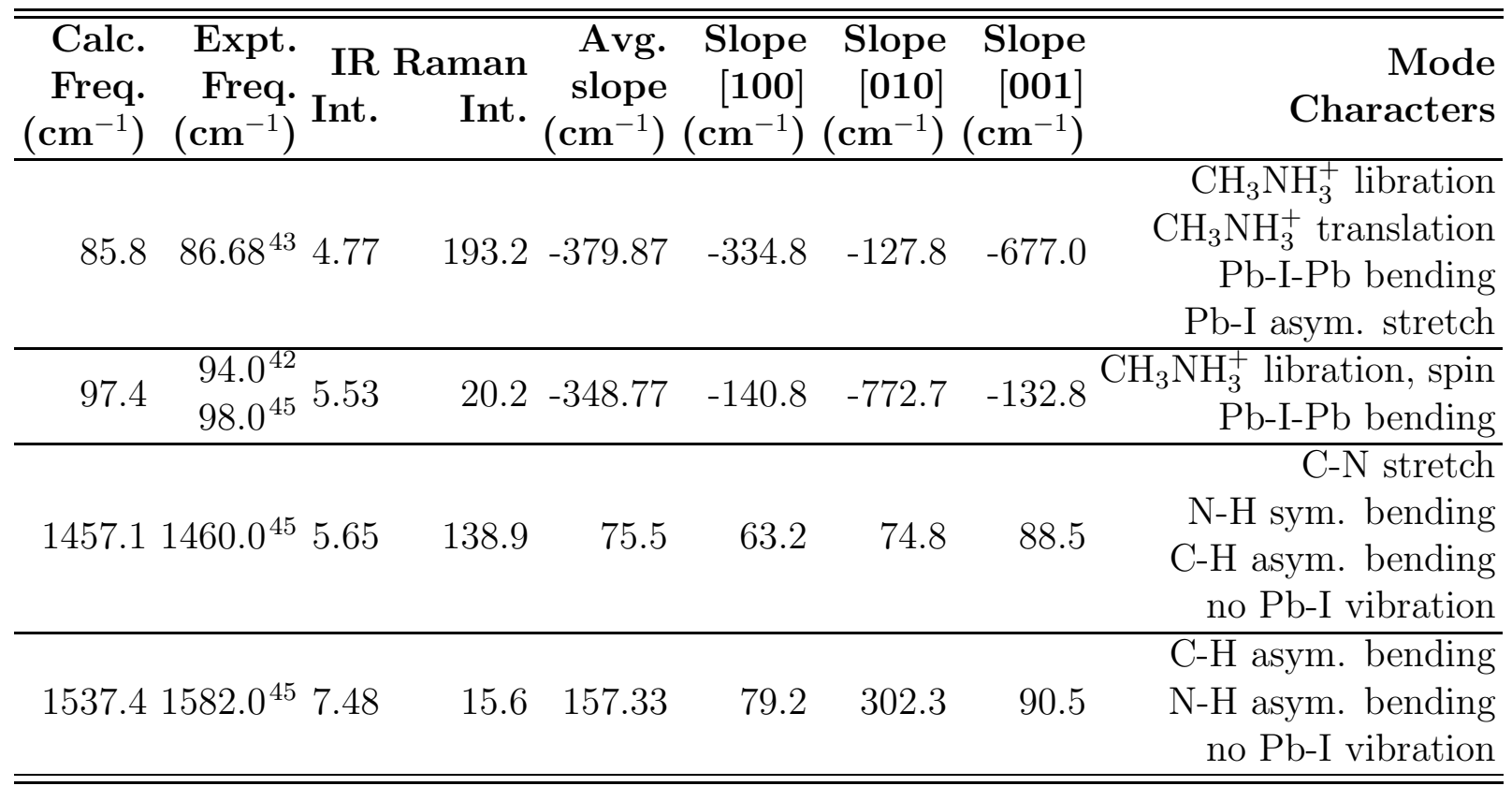

${ }^{a}$ Absolute IR intensity is in $(\mathrm{D} / \AA)^{2} / \mathrm{amu}$ and Raman intensity in $\AA^{4} / \mathrm{amu}$.

$\mathrm{cm}^{-1}$ which is well within the expected shifts from $1 \%$ strain in case of our cubic structure. One contribution to the temperature-dependent frequency shift is thermal expansion which is a sign of significant effect of anharmonicity. Our results show that this is a relatively minor contribution because our predicted shifts due to thermal expansion are much less than the shifts reported by Nakada et al., $\underline{\underline{45}}$ in accordance with results of Bonini et al. for graphite. $\underline{\underline{97}}$

Finally, note that the deviation between calculated and measured frequencies for these modes is larger than expected strain shifts. Due to this unavoidable systematic difference, we would recommend use of our calculated slopes to infer relative strains in a sample, rather than by direct comparison of experimental frequencies to our calculated frequencies. $\underline{61}$

\subsection{Calculation of mode Grüneisen parameter}

The uniaxial mode Grüneisen parameter is calculated using the slope of the frequency vs strain curve for each mode (Fig. 10), as done in a-Si. $\underline{61} \mathrm{~Pb}-\mathrm{I}$ vibrations at lower frequency have significant values, whereas $\mathrm{CH}_{3} \mathrm{NH}_{3}^{+}$ion vibrations at higher frequency have much 
smaller values. One of the reasons for the low values at high frequency is the high frequency itself, as we divide the slope with the mode frequency, although some high-frequency modes do have large absolute slopes. To connect to macroscopic properties, we calculate the Grüneisen parameter, as the weighted average over all the modes using the Bose-Einstein formula for heat capacity. In distinction to F. Brivio et al., $\underline{\underline{41}}$ we are using uniaxial strain, and we include only modes at $q=\Gamma$, because of the conceptual problem in the quasiharmonic approximation of how to handle the imaginary frequencies. $\frac{98}{}$ Also, due to dynamical disorder $\stackrel{49}{ }$, which is not included in our calculation, the phonon bandstructure away from $q=\Gamma$ may be less accurate or lose some of its meaning without periodicity. The imaginary frequencies are indicative of the fact that the cubic structure is not the most stable phase at $T=0$, and were observed in previous work. $\underline{\underline{41}}$ They occur around $q=\mathrm{R}$ and $q=\mathrm{M}$, and are not reduced by strain (Table S6). Our calculated values of the directional Grüneisen parameter at $330 \mathrm{~K}$ (the transition temperature to tetragonal) for strain along [100], [010], and [001] are $1.06,2.10$, and -0.51 , respectively, for an average of 0.88 . The isotropic value reported by F. Brivio et al., $\underline{41}$ averaged over temperature, was 1.6. We expect the difference is due to the handling of Brillouin zone integration, as well as their calculation via quasiharmonic thermal expansion rather than mode Grüneisen parameters. This result shows a directional dependency of the Grüneisen parameter that suggests an unusual negative thermal expansion along [001] direction for cubic MAPI, due to the negative value. While the existence of cation rotations at $330 \mathrm{~K}$ complicates the physical interpretation of this result, it connects to previous studies reporting that tetragonal perovskites have a negative thermal expansion coefficient along the [001] or $c$-axis, $\stackrel{52,53}{,}$ which is perpendicular to the direction of polarization of the $\mathrm{CH}_{3} \mathrm{NH}_{3}^{+}$ion and also the largest lattice vector, in both cubic and tetragonal structures. 


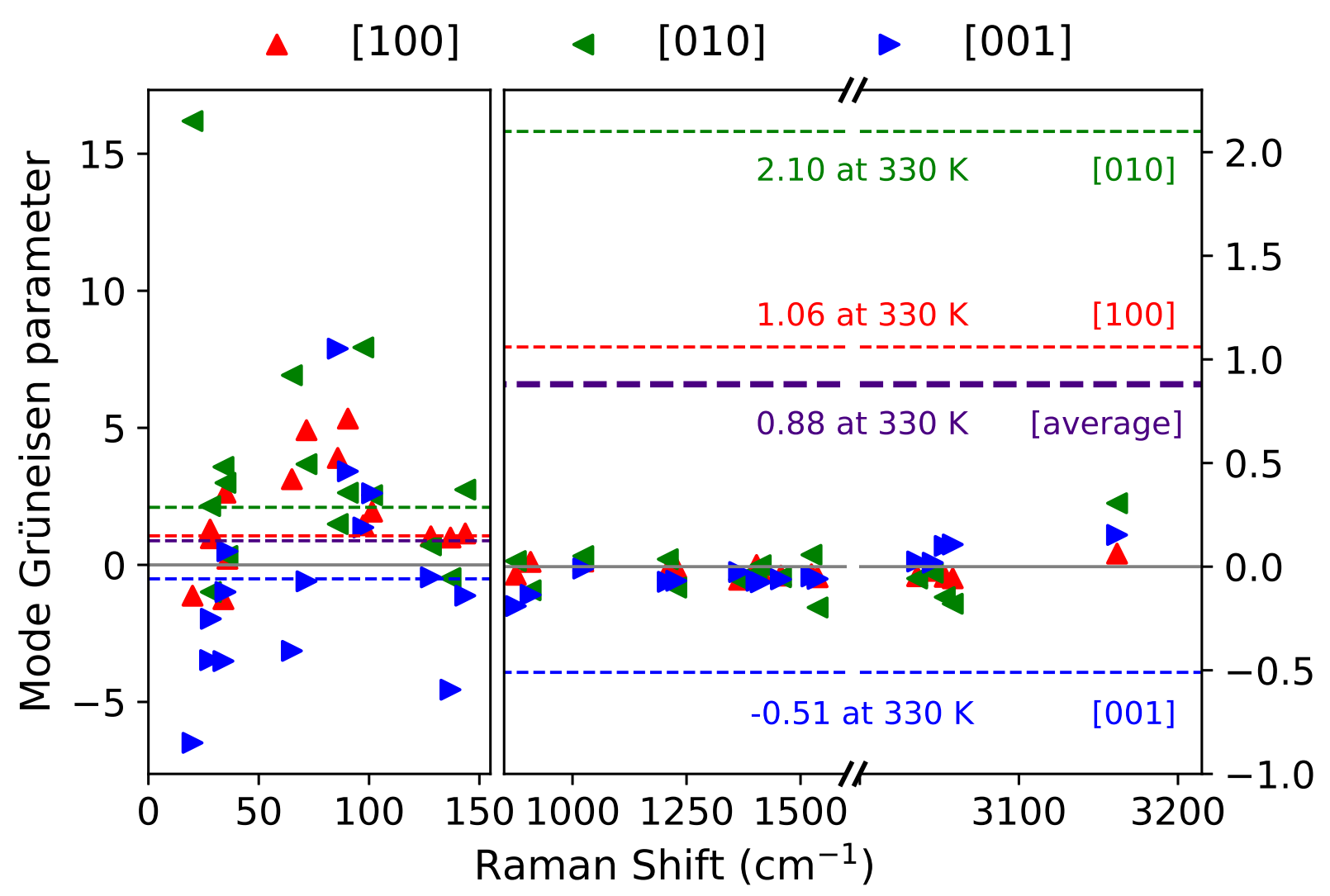

Figure 10: Calculated uniaxial mode Grüneisen parameters using slopes of the frequency vs strain graph for each mode in three different crystallographic directions. Dashed lines in red, green and blue represent Grüneisen parameter calculated at $330 \mathrm{~K}$ for different uniaxial directions. Thick dashed line in violet represents the average Grüneisen parameter.

\section{Conclusion}

We have comprehensively studied the structural and vibrational properties of cubic $\mathrm{CH}_{3} \mathrm{NH}_{3} \mathrm{PbI}_{3}$ under uniaxial strain. By analysing the dynamical matrix of the system under each strain we are able to identify the interactions which contribute significantly to the frequency changes due to applied strain. The phonon modes have frequency changes under strain, which can show linear, parabolic, or irregular patterns. Linear is associated with change in dynamical matrix but no change in mode eigenvector with strain, while parabolic is associated with changes in both dynamical matrix and mode eigenvector, in accordance with a perturbative analysis. Irregular patterns are due to large changes in mode eigenvector due to discontin- 
uous structural changes, which are a sign of anharmonicity. These changes give insight into the interplay between structure, strain, and vibrations, and show approximate symmetries for some modes. We find that not only the $\mathrm{Pb}-\mathrm{I}$ bond lengths but also the $\mathrm{Pb}-\mathrm{I}-\mathrm{Pb}$ bond angles change under strain, showing a buckling of the $\mathrm{Pb}-\mathrm{I}$ lattice. Decrease in $\mathrm{N}-\mathrm{H}$ bond length under compressive strain may be connected to the increased stability under compressive strain. We also find that the cation rotates with respect to the lattice under strain. We have identified 4 modes that are promising for IR or Raman microscopy measurement of local strain, as done in other semiconductors and even amorphous Si which has broad peaks. Results on the mode Grüneisen parameters and macroscopic Grüneisen parameters give insight into anharmonicity and directional negative thermal expansion. Our study of the cubic phase is relevant not only to high temperatures but also to cubic phases stabilized by ligands or other cations. Our results are expected to be similar in many respects to

results for the tetragonal and orthorhombic phases, particularly in the mid-frequency range where the spectra are similar, and these phases will be the subject of future publications. We look forward to experimental work to confirm these results with uniform strain, and employ our calibration for strain mapping. Our work opens the for a standard bench-top characterization method to be usable for analyzing the critical role of local strain in hybrid perovskite photovoltaics.

\section{Supporting Information Available}

Structural parameters, bandstructure and bandgap, phonon mode characterization, structural changes with strain, mode eigenvector and frequency changes with strain, imaginary modes at the $q=\mathrm{M}$ and $q=\mathrm{R}$ points $(\mathrm{PDF})$. Relaxed atomic coordinates at each strain including phonon eigenvectors (AXSF). 


\section{Acknowledgement}

We acknowledge a helpful discussion with Yong Zhang. Work was supported by UC Merced start-up funds and the Merced nAnomaterials Center for Energy and Sensing (MACES), a NASA-funded research and education center, under award NNX15AQ01. This work used computational resources from the Multi-Environment Computer for Exploration and Discovery (MERCED) cluster at UC Merced, funded by National Science Foundation Grant No. ACI-1429783, and the National Energy Research Scientific Computing Center (NERSC), a U.S. Department of Energy Office of Science User Facility operated under Contract No. DE-AC02-05CH11231.

\section{References}

(1) Green, M. A.; Ho-Baillie, A.; Snaith, H. J. The emergence of perovskite solar cells. Nat. Photon. 2014, 8, 506-514.

(2) Frohna, K.; Deshpande, T.; Harter, J.; Peng, W.; Barker, B. A.; Neaton, J. B.; Louie, S. G.; Bakr, O. M.; Hsieh, D.; Bernardi, M. Inversion symmetry and bulk Rashba effect in methylammonium lead iodide perovskite single crystals. Nat. Commun. 2018, 9, 1829.

(3) Umari, P.; Mosconi, E.; De Angelis, F. Relativistic GW calculations on $\mathrm{CH}_{3} \mathrm{NH}_{3} \mathrm{PbI}_{3}$ and $\mathrm{CH}_{3} \mathrm{NH}_{3} \mathrm{SnI}_{3}$ perovskites for solar cell applications. Sci. Rep. 2014, 4, 4467.

(4) Shirayama, M.; Kadowaki, H.; Miyadera, T.; Sugita, T.; Tamakoshi, M.; Kato, M.; Fujiseki, T.; Murata, D.; Hara, S.; Murakami, T. N. et al. Optical transitions in hybrid perovskite solar cells: Ellipsometry, density functional theory, and quantum efficiency analyses for $\mathrm{CH}_{3} \mathrm{NH}_{3} \mathrm{PbI}_{3}$. Phys. Rev. Appl. 2016, 5, 014012 .

(5) Stranks, S. D.; Eperon, G. E.; Grancini, G.; Menelaou, C.; Alcocer, M. J. P.; Leijtens, T.; Herz, L. M.; Petrozza, A.; Snaith, H. J. Electron-hole diffusion lengths ex- 
ceeding 1 micrometer in an organometal trihalide perovskite absorber. Science $\mathbf{2 0 1 3}$, 342, 341-344.

(6) Xing, G.; Mathews, N.; Sun, S.; Lim, S. S.; Lam, Y. M.; Grätzel, M.; Mhaisalkar, S.; Sum, T. C. Long-range balanced electron- and hole-transport lengths in organicinorganic $\mathrm{CH}_{3} \mathrm{NH}_{3} \mathrm{PbI}_{3}$. Science 2013, 342, 344-347.

(7) Wehrenfennig, C.; Eperon, G. E.; Johnston, M. B.; Snaith, H. J.; Herz, L. M. High charge carrier mobilities and lifetimes in organolead trihalide perovskites. Adv. Mater. 2014, 26, 1584-1589.

(8) Kojima, A.; Teshima, K.; Shirai, Y.; Miyasaka, T. Organometal halide perovskites as visible-light sensitizers for photovoltaic cells. J. Am. Chem. Soc 2009, 131, 6050-6051.

(9) Best Research-Cell Efficiency Chart from National Renewable Energy Laboratory. https://www.nrel.gov/pv/assets/pdfs/best-research-cell-efficiencies.20200708.pdf (accessed September 27, 2020).

(10) Eperon, G. E.; Leijtens, T.; Bush, K. A.; Prasanna, R.; Green, T.; Wang, J. T.-W.; McMeekin, D. P.; Volonakis, G.; Milot, R. L.; May, R. et al. Perovskite-perovskite tandem photovoltaics with optimized band gaps. Science 2016, 354, 861-865.

(11) Lin, Q.; Armin, A.; Nagiri, R. C. R.; Burn, P. L.; Meredith, P. Electro-optics of perovskite solar cells. Nat. Photon. 2015, 9, 106.

(12) Yang, D.; Yang, R.; Priya, S.; Liu, S. F. Recent advances in flexible perovskite solar cells: Fabrication and applications. Angew. Chem. Int. Ed. 2019, 58, 4466-4483.

(13) Bailie, C. D.; Christoforo, M. G.; Mailoa, J. P.; Bowring, A. R.; Unger, E. L.; Nguyen, W. H.; Burschka, J.; Pellet, N.; Lee, J. Z.; Grätzel, M. et al. Semi-transparent perovskite solar cells for tandems with silicon and CIGS. Energy Environ. Sci 2015, 8, $956-963$. 
(14) Roldán-Carmona, C.; Malinkiewicz, O.; Soriano, A.; Espallargas, G. M.; Garcia, A.; Reinecke, P.; Kroyer, T.; Dar, M. I.; Nazeeruddin, M. K.; Bolink, H. J. Flexible high efficiency perovskite solar cells. Energy Environ. Sci 2014, 7, 994-997.

(15) Ping, Y.; Zhang, J. Z. Spin-optotronic properties of organometal halide perovskites. J. Phys. Chem. Lett. 2018, 9, 6103-6111.

(16) Nikolaidou, K.; Sarang, S.; Hoffman, C.; Mendewala, B.; Ishihara, H.; Lu, J. Q.; Ilan, B.; Tung, V.; Ghosh, S. Hybrid perovskite thin films as highly efficient luminescent solar concentrators. Adv. Opt. Mater. 2016, 4, 2126-2132.

(17) Kim, H.; Zhao, L.; Price, J. S.; Grede, A. J.; Roh, K.; Brigeman, A. N.; Lopez, M.; Rand, B. P.; Giebink, N. C. Hybrid perovskite light-emitting diodes under intense electrical excitation. Nat. Commun. 2018, 9, 4893.

(18) Niu, G.; Guo, X.; Wang, L. Review of recent progress in chemical stability of perovskite solar cells. J. Mater. Chem. A 2015, 3, 8970-8980.

(19) Conings, B.; Drijkoningen, J.; Gauquelin, N.; Babayigit, A.; D'Haen, J.; D’Olieslaeger, L.; Ethirajan, A.; Verbeeck, J.; Manca, J.; Mosconi, E. et al. Intrinsic thermal instability of methylammonium lead trihalide perovskite. Adv. Energy Mater. 2015, 5, 1500477.

(20) Joshi, P. H.; Zhang, L.; Hossain, I. M.; Abbas, H. A.; Kottokkaran, R.; Nehra, S. P.; Dhaka, M.; Noack, M.; Dalal, V. L. The physics of photon induced degradation of perovskite solar cells. AIP Adv. 2016, 6, 115114.

(21) Lee, J.-W.; Kim, D.-H.; Kim, H.-S.; Seo, S.-W.; Cho, S. M.; Park, N.-G. Formamidinium and cesium hybridization for photo-and moisture-stable perovskite solar cell. Adv. Energy Mater. 2015, 5, 1501310. 
(22) Li, X.; Tschumi, M.; Han, H.; Babkair, S. S.; Alzubaydi, R. A.; Ansari, A. A.; Habib, S. S.; Nazeeruddin, M. K.; Zakeeruddin, S. M.; Grätzel, M. Outdoor performance and stability under elevated temperatures and long-term light soaking of triple-layer mesoporous perovskite photovoltaics. Energy Technol. 2015, 3, 551-555.

(23) Ong, K. P.; Goh, T. W.; Xu, Q.; Huan, A. Structural evolution in methylammonium lead iodide $\mathrm{CH}_{3} \mathrm{NH}_{3} \mathrm{PbI}_{3}$. J. Phys. Chem. A 2015, 119, 11033-11038.

(24) Nie, W.; Blancon, J. C.; Neukirch, A. J.; Appavoo, K.; Tsai, H.; Chhowalla, M.; Alam, M. A.; Sfeir, M. Y.; Katan, C.; Even, J. et al. Light-activated photocurrent degradation and self-healing in perovskite solar cells. Nat. Commun. 2016, 7, 1-9.

(25) Zhao, J.; Deng, Y.; Wei, H.; Zheng, X.; Yu, Z.; Shao, Y.; Shield, J. E.; Huang, J. Strained hybrid perovskite thin films and their impact on the intrinsic stability of perovskite solar cells. Sci. Adv. 2017, 3, eaao5616.

(26) Faghihnasiri, M.; Izadifard, M.; Ghazi, M. E. DFT study of mechanical properties and stability of cubic methylammonium lead halide perovskites $\left(\mathrm{CH}_{3} \mathrm{NH}_{3} \mathrm{PbI}_{3}, \mathrm{X}=\mathrm{I}, \mathrm{Br}\right.$, Cl). J. Phys. Chem. C 2017, 121, 27059-27070.

(27) Zhang, L.; Geng, W.; Tong, C. J.; Chen, X.; Cao, T.; Chen, M. Strain induced electronic structure variation in methyl-ammonium lead iodide perovskite. Sci. Rep. 2018, 8, 7760 .

(28) Bechtel, J. S.; Van der Ven, A. Octahedral tilting instabilities in inorganic halide perovskites. Phys. Rev. Mater. 2018, 2, 025401.

(29) Tsai, H.; Asadpour, R.; Blancon, J.-C.; Stoumpos, C. C.; Durand, O.; Strzalka, J. W.; Chen, B.; Verduzco, R.; Ajayan, P. M.; Tretiak, S. et al. Light-induced lattice expansion leads to high-efficiency perovskite solar cells. Science 2018, 360, 67-70. 
(30) Jones, T. W.; Osherov, A.; Alsari, M.; Sponseller, M.; Duck, B. C.; Jung, Y.-K.; Settens, C.; Niroui, F.; Brenes, R.; Stan, C. V. et al. Lattice strain causes non-radiative losses in halide perovskites. Energy Environ. Sci 2019, 12, 596-606.

(31) Zhu, C.; Niu, X.; Fu, Y.; Li, N.; Hu, C.; Chen, Y.; He, X.; Na, G.; Liu, P.; Zai, H. et al. Strain engineering in perovskite solar cells and its impacts on carrier dynamics. Nat. Commun. 2019, 10, 1-11.

(32) Slotcavage, D. J.; Karunadasa, H. I.; McGehee, M. D. Light-induced phase segregation in halide-perovskite absorbers. ACS Energy Lett. 2016, 1, 1199-1205.

(33) Chen, Y.; Lei, Y.; Li, Y.; Yu, Y.; Cai, J.; Chiu, M.-H.; Rao, R.; Gu, Y.; Wang, C.; Choi, W. et al. Strain engineering and epitaxial stabilization of halide perovskites. Nature 2020, 577, 209-215.

(34) Huang, J.; Yuan, Y.; Shao, Y.; Yan, Y. Understanding the physical properties of hybrid perovskites for photovoltaic applications. Nat. Rev. Mater. 2017, 2, 17042.

(35) Jena, A. K.; Kulkarni, A.; Miyasaka, T. Halide perovskite photovoltaics: background, status, and future prospects. Chem. Rev. 2019, 119, 3036-3103.

(36) Even, J. Pedestrian guide to symmetry properties of the reference cubic structure of 3D all-inorganic and hybrid perovskites. J. Phys. Chem. Lett. 2015, 6, 2238-2242.

(37) Sarang, S.; Bonabi Naghadeh, S.; Luo, B.; Kumar, P.; Betady, E.; Tung, V.; Scheibner, M.; Zhang, J. Z.; Ghosh, S. Stabilization of the cubic crystalline phase in organometal halide perovskite quantum dots via surface energy manipulation. J. Phys. Chem. Lett. 2017, 8, 5378-5384.

(38) Wu, C.; Chen, K.; Guo, D.; Wang, S.; Li, P. Cations substitution tuning phase stability in hybrid perovskite single crystals by strain relaxation. $R S C A d v$. 2018, 8, 2900-2905. 
(39) Sheng, R.; Ho-Baillie, A.; Huang, S.; Chen, S.; Wen, X.; Hao, X.; Green, M. A. Methylammonium lead bromide perovskite-based solar cells by vapor-assisted deposition. $J$. Phys. Chem. C 2015, 119, 3545-3549.

(40) Zhang, H.; Qiao, X.; Shen, Y.; Moehl, T.; Zakeeruddin, S. M.; Grätzel, M.; Wang, M. Photovoltaic behaviour of lead methylammonium triiodide perovskite solar cells down to 80 K. J. Mater. Chem. A 2015, 3, 11762-11767.

(41) Brivio, F.; Frost, J. M.; Skelton, J. M.; Jackson, A. J.; Weber, O. J.; Weller, M. T.; Goñi, A. R.; Leguy, A. M. A.; Barnes, P. R. F.; Walsh, A. Lattice dynamics and vibrational spectra of the orthorhombic, tetragonal, and cubic phases of methylammonium lead iodide. Phys. Rev. B 2015, 92, 144308.

(42) Luan, M.; Song, J.; Wei, X.; Chen, F.; Liu, J. Controllable growth of bulk cubic-phase $\mathrm{CH}_{3} \mathrm{NH}_{3} \mathrm{PbI}_{3}$ single crystal with exciting room-temperature stability. CrystEngComm 2016, 18, 5257-5261.

(43) Qiu, J.; McDowell, L. L.; Shi, Z. Room-temperature cubic perovskite thin films by three-step all-vapor conversion from PbSe to $\mathrm{MAPbI}_{3}$. Cryst. Growth Des. 2019, 19, 2001-2009.

(44) Ledinský, M.; Löper, P.; Niesen, B.; Holovský, J.; Moon, S.-J.; Yum, J.-H.; De Wolf, S.; Fejfar, A.; Ballif, C. Raman spectroscopy of organic-inorganic halide perovskites. J. Phys. Chem. Lett. 2015, 6, 401-406.

(45) Nakada, K.; Matsumoto, Y.; Shimoi, Y.; Yamada, K.; Furukawa, Y. Temperaturedependent evolution of Raman spectra of methylammonium lead halide perovskites, $\mathrm{CH}_{3} \mathrm{NH}_{3} \mathrm{PbI}_{3}(\mathrm{X}=\mathrm{I}, \mathrm{Br})$. Molecules 2019, 24, 626 .

(46) Pérez-Osorio, M. A.; Lin, Q.; Phillips, R. T.; Milot, R. L.; Herz, L. M.; Johnston, M. B.; Giustino, F. Raman spectrum of the organic-inorganic halide perovskite $\mathrm{CH}_{3} \mathrm{NH}_{3} \mathrm{PbI}_{3}$ 
from first principles and high-resolution low-temperature Raman measurements. $J$. Phys. Chem. C 2018, 122, 21703-21717.

(47) Pérez-Osorio, M. A.; Milot, R. L.; Filip, M. R.; Patel, J. B.; Herz, L. M.; Johnston, M. B.; Giustino, F. Vibrational properties of the organic-inorganic halide perovskite $\mathrm{CH}_{3} \mathrm{NH}_{3} \mathrm{PbI}_{3}$ from theory and experiment: factor group analysis, first-principles calculations, and low-temperature infrared spectra. J. Phys. Chem. C 2015, 119, 25703-25718.

(48) Glaser, T.; Müller, C.; Sendner, M.; Krekeler, C.; Semonin, O. E.; Hull, T. D.; Yaffe, O.; Owen, J. S.; Kowalsky, W.; Pucci, A. et al. Infrared spectroscopic study of vibrational modes in methylammonium lead halide perovskites. J. Phys. Chem. Lett. 2015, 6, 2913-2918.

(49) Leguy, A. M.; Goñi, A. R.; Frost, J. M.; Skelton, J.; Brivio, F.; Rodríguez-Martínez, X.; Weber, O. J.; Pallipurath, A.; Alonso, M. I.; Campoy-Quiles, M. et al. Dynamic disorder, phonon lifetimes, and the assignment of modes to the vibrational spectra of methylammonium lead halide perovskites. Phys. Chem. Chem. Phys. 2016, 18, 2705127066 .

(50) Chen, Q.; Liu, H.; Kim, H.-S.; Liu, Y.; Yang, M.; Yue, N.; Ren, G.; Zhu, K.; Liu, S.; Park, N.-G. et al. Multiple-stage structure transformation of organic-inorganic hybrid perovskite $\mathrm{CH}_{3} \mathrm{NH}_{3} \mathrm{PbI}_{3}$. Phys. Rev. $X$ 2016, 6, 031042 .

(51) Matsuishi, K.; Ishihara, T.; Onari, S.; Chang, Y.; Park, C. Optical properties and structural phase transitions of lead-halide based inorganic-organic 3D and 2D perovskite semiconductors under high pressure. phys. stat. sol. (b) 2004, 241, 3328-3333.

(52) Ge, C.; Hu, M.; Wu, P.; Tan, Q.; Chen, Z.; Wang, Y.; Shi, J.; Feng, J. Ultralow thermal conductivity and ultrahigh thermal expansion of single-crystal organic-inorganic hybrid perovskite $\mathrm{CH}_{3} \mathrm{NH}_{3} \mathrm{PbX}_{3}(\mathrm{X}=\mathrm{Cl}, \mathrm{Br}, \mathrm{I})$. J. Phys. Chem. C 2018, 122, 15973-15978. 
(53) Heiderhoff, R.; Haeger, T.; Pourdavoud, N.; Hu, T.; Al-Khafaji, M.; Mayer, A.; Chen, Y.; Scheer, H.-C.; Riedl, T. Thermal conductivity of methylammonium lead halide perovskite single crystals and thin films: A comparative study. J. Phys. Chem. C 2017, 121, 28306-28311.

(54) Anastassakis, E.; Pinczuk, A.; Burstein, E.; Pollak, F.; Cardona, M. Effect of static uniaxial stress on the Raman spectrum of silicon. Solid State Commun. 1993, 88, 1053-1058.

(55) Fabini, D. H.; Seshadri, R.; Kanatzidis, M. G. The underappreciated lone pair in halide perovskites underpins their unusual properties. MRS Bull. 2020, 45, 467-477.

(56) Zhu, T.; Ertekin, E. Mixed phononic and non-phononic transport in hybrid lead halide perovskites: glass-crystal duality, dynamical disorder, and anharmonicity. Energy Environ. Sci. 2019, 12, 216-229.

(57) Rolston, N.; Bush, K. A.; Printz, A. D.; Gold-Parker, A.; Ding, Y.; Toney, M. F.; McGehee, M. D.; Dauskardt, R. H. Engineering stress in perovskite solar cells to improve stability. Adv. Energy Mater. 2018, 8, 1802139.

(58) Pistor, P.; Ruiz, A.; Cabot, A.; Izquierdo-Roca, V. Advanced Raman spectroscopy of methylammonium lead iodide: Development of a non-destructive characterisation methodology. Sci. Rep. 2016, 6, 35973.

(59) De Wolf, I. Micro-Raman spectroscopy to study local mechanical stress in silicon integrated circuits. Semicond. Sci. Technol. 1996, 11, 139.

(60) Rao, R.; Islam, A. E.; Singh, S.; Berry, R.; Kawakami, R. K.; Maruyama, B.; Katoch, J. Spectroscopic evaluation of charge-transfer doping and strain in graphene/MoS $\mathrm{M}_{2}$ heterostructures. Phys. Rev. B 2019, 99, 195401. 
(61) Strubbe, D. A.; Johlin, E. C.; Kirkpatrick, T. R.; Buonassisi, T.; Grossman, J. C. Stress effects on the Raman spectrum of an amorphous material: Theory and experiment on a-Si:H. Phys. Rev. B 2015, 92, 241202(R).

(62) Su, W.; Kumar, N.; Dai, N.; Roy, D. Nanoscale mapping of intrinsic defects in singlelayer graphene using tip-enhanced Raman spectroscopy. Chem. Commun. 2016, 52, $8227-8230$.

(63) Lyu, B.; Li, H.; Jiang, L.; Shan, W.; Hu, C.; Deng, A.; Ying, Z.; Wang, L.; Zhang, Y.; Bechtel, H. A. et al. Phonon polariton-assisted infrared nanoimaging of local strain in hexagonal boron nitride. Nano Lett. 2019, 19, 1982-1989.

(64) Smith, K.; Nowadnick, E.; Fan, S.; Khatib, O.; Lim, S. J.; Gao, B.; Harms, N.; Neal, S.; Kirkland, J.; Martin, M. et al. Infrared nano-spectroscopy of ferroelastic domain walls in hybrid improper ferroelectric $\mathrm{Ca}_{3} \mathrm{Ti}_{2} \mathrm{O}_{7}$. Nat. Commun. 2019, 10, 1-9.

(65) Baroni, S.; de Gironcoli, S.; Dal Corso, A.; Giannozzi, P. Phonons and related crystal properties from density-functional perturbation theory. Rev. Mod. Phys. 2001, 73, 515562.

(66) Giannozzi, P.; Andreussi, O.; Brumme, T.; Bunau, O.; Nardelli, M. B.; Calandra, M.; Car, R.; Cavazzoni, C.; Ceresoli, D.; Cococcioni, M. et al. Advanced capabilities for materials modelling with Quantum ESPRESSO. J. Phys.: Condens. Matt. 2017, 29, 465901.

(67) Giannozzi, P.; Baroni, S.; Bonini, N.; Calandra, M.; Car, R.; Cavazzoni, C.; Ceresoli, D.; Chiarotti, G. L.; Cococcioni, M.; Dabo, I. et al. Quantum ESPRESSO: a modular and open-source software project for quantum simulations of materials. J. Phys.: Condens. Matt. 2009, 21, 395502.

(68) Baroni, S.; Dal Corso, A.; De Gironcoli, S.; Giannozzi, P.; Cavazzoni, C.; Ballabio, G.; Scandolo, S.; Chiarotti, G.; Focher, P.; Pasquarello, A. et al. Quantum ESPRESSO: 
open-source package for research in electronic structure, simulation, and optimization. Code available from http://www.quantum-espresso.org 2005

(69) Perdew, J. P.; Zunger, A. Self-interaction correction to density-functional approximations for many-electron systems. Phys. Rev. B 1981, 23, 5048-5079.

(70) Perdew, J. P.; Wang, Y. Accurate and simple analytic representation of the electron-gas correlation energy. Phys. Rev. B 1992, 45, 13244.

(71) Hamann, D. R. Optimized norm-conserving Vanderbilt pseudopotentials. Phys. Rev. B 2013, 88, 085117.

(72) Van Setten, M.; Giantomassi, M.; Bousquet, E.; Verstraete, M. J.; Hamann, D. R.; Gonze, X.; Rignanese, G.-M. The PseudoDojo: Training and grading a 85 element optimized norm-conserving pseudopotential table. Comput. Phys. Commun. 2018, 226, $39-54$.

(73) The PseudoDojo: Training and grading a 85 element optimized norm-conserving pseudopotential table. http://www.pseudo-dojo.org (accessed February 09, 2020).

(74) Initial structure of MAPI. https://github.com/WMD-group/hybrid-perovskites/blob/master/20 (accessed February 09, 2020).

(75) Weller, M. T.; Weber, O. J.; Henry, P. F.; Di Pumpo, A. M.; Hansen, T. C. Complete structure and cation orientation in the perovskite photovoltaic methylammonium lead iodide between 100 and 352 K. Chem. Commun. 2015, 51, 4180-4183.

(76) Baikie, T.; Fang, Y.; Kadro, J. M.; Schreyer, M.; Wei, F.; Mhaisalkar, S. G.; Graetzel, M.; White, T. J. Synthesis and crystal chemistry of the hybrid perovskite $\mathrm{CH}_{3} \mathrm{NH}_{3} \mathrm{PbI}_{3}$ for solid-state sensitised solar cell applications. J. Mater. Chem. A 2013, $1,5628-5641$. 
(77) Stoumpos, C. C.; Malliakas, C. D.; Kanatzidis, M. G. Semiconducting tin and lead iodide perovskites with organic cations: phase transitions, high mobilities, and nearinfrared photoluminescent properties. Inorg. Chem. 2013, 52, 9019-9038.

(78) Leguy, A. M.; Frost, J. M.; McMahon, A. P.; Sakai, V. G.; Kockelmann, W.; Law, C.; Li, X.; Foglia, F.; Walsh, A.; O'Regan, B. C. et al. The dynamics of methylammonium ions in hybrid organic-inorganic perovskite solar cells. Nat. Commun. 2015, 6, 7124.

(79) Whalley, L. D.; Frost, J. M.; Jung, Y.-K.; Walsh, A. Perspective: Theory and simulation of hybrid halide perovskites. J. Chem. Phys. 2017, 146, 220901.

(80) McKechnie, S.; Frost, J. M.; Pashov, D.; Azarhoosh, P.; Walsh, A.; Van Schilfgaarde, M. Dynamic symmetry breaking and spin splitting in metal halide perovskites. Phys. Rev. B 2018, 98, 085108 .

(81) Giorgi, G.; Fujisawa, J.-i. I.; Segawa, H.; Yamashita, K. Small photocarrier effective masses featuring ambipolar transport in methylammonium lead iodide perovskite: A density functional analysis. J. Phys. Chem. Lett. 2013, 4, 4213-4216.

(82) Perdew, J. P.; Burke, K.; Ernzerhof, M. Generalized gradient approximation made simple. Phys. Rev. Lett. 1996, 77, 3865-3868.

(83) Perdew, J. P.; Ruzsinszky, A.; Csonka, G. I.; Vydrov, O. A.; Scuseria, G. E.; Constantin, L. A.; Zhou, X.; Burke, K. Restoring the density-gradient expansion for exchange in solids and surfaces. Phys. Rev. Lett. 2008, 100, 136406.

(84) Parker Jr, J.; Feldman, D.; Ashkin, M. Raman scattering by silicon and germanium. Phys. Rev. 1967, 155, 712.

(85) Anastassakis, E.; Cantarero, A.; Cardona, M. Piezo-Raman measurements and anharmonic parameters in silicon and diamond. Phys. Rev. B 1990, 41, 7529. 
(86) Giustino, F. Materials modelling using density functional theory: properties and predictions; Oxford University Press, 2014.

(87) Feng, J. Mechanical properties of hybrid organic-inorganic $\mathrm{CH}_{3} \mathrm{NH}_{3} \mathrm{BX}$ ( $\mathrm{B}=\mathrm{Sn}, \mathrm{Pb}$; $\mathrm{X}=\mathrm{Br}, \mathrm{I})$ perovskites for solar cell absorbers. APL Mater. 2014, 2, 081801.

(88) Lazzeri, M.; Mauri, F. First-principles calculation of vibrational Raman spectra in large systems: Signature of small rings in crystalline $\mathrm{SiO}_{2}$. Phys. Rev. Lett. 2003, 90, 036401.

(89) Mounet, N. Structural, vibrational and thermodynamic properties of carbon allotropes from first-principles: diamond, graphite, and nanotubes. Masters Thesis, Massachusetts Institute of Technology $\mathbf{2 0 0 5}$

(90) Vočadlo, N. L.; Price, G. D. The Grüneisen parameter - computer calculations via lattice dynamics. Phys. Earth Planet. Inter. 1994, 82, 261-270.

(91) Fennie, C. J.; Rabe, K. M. Structural and dielectric properties of $\mathrm{Sr}_{2} \mathrm{TiO}_{4}$ from first principles. Phys. Rev. B 2003, 68, 184111.

(92) Ganesan, S.; Maradudin, A.; Oitmaa, J. A lattice theory of morphic effects in crystals of the diamond structure. Ann. Phys. 1970, 56, 556-594.

(93) Xue, D.-J.; Hou, Y.; Liu, S.-C.; Wei, M.; Chen, B.; Huang, Z.; Li, Z.; Sun, B.; Proppe, A. H.; Dong, Y. et al. Regulating strain in perovskite thin films through chargetransport layers. Nat. Commun. 2020, 11, 1-8.

(94) Rappich, J.; Lang, F.; Brus, V. V.; Shargaieva, O.; Dittrich, T.; Nickel, N. H. Lightinduced defect generation in $\mathrm{CH}_{3} \mathrm{NH}_{3} \mathrm{PbI}_{3}$ thin films and single crystals. Solar $R R L$ 2020, 4, 1900216.

(95) Kang, B.; Biswas, K. Preferential $\mathrm{CH}_{3} \mathrm{NH}_{3}^{+}$alignment and octahedral tilting affect charge localization in cubic phase $\mathrm{CH}_{3} \mathrm{NH}_{3} \mathrm{PbI}_{3}$. J. Phys. Chem. C 2017, 121, 83198326. 
(96) Wu, W.; Wang, J.; Ercius, P.; Wright, N. C.; Leppert-Simenauer, D. M.; Burke, R. A.; Dubey, M.; Dogare, A. M.; Pettes, M. T. Giant mechano-optoelectronic effect in an atomically thin semiconductor. Nano Lett. 2018, 18, 2351-2357.

(97) Bonini, N.; Lazzeri, M.; Marzari, N.; Mauri, F. Phonon anharmonicities in graphite and graphene. Phys. Rev. Lett. 2007, 99, 176802.

(98) Deringer, V. L.; Stoffel, R. P.; Dronskowski, R. Vibrational and thermodynamic properties of GeSe in the quasiharmonic approximation. Phys. Rev. B 2014, 89, 094303.

\section{TOC Graphic}

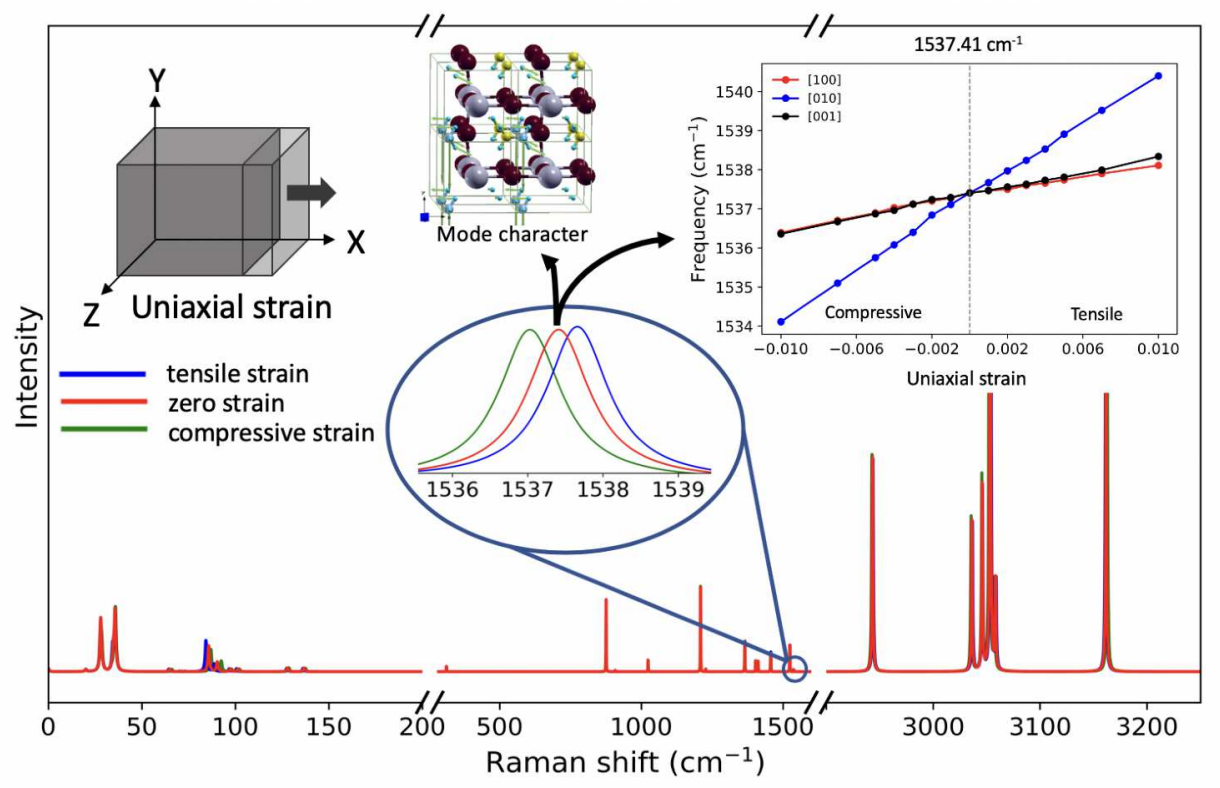

